\title{
hsa_circ_0058357 acts as a ceRNA to promote non-small cell lung cancer progression via the hsa-miR-24-3p/AVL9 axis
}

\author{
DONGSHAN WEI ${ }^{1 *}$, LIFANG SUN ${ }^{2,3^{*}}$ and WEI FENG ${ }^{4-6}$ \\ ${ }^{1}$ Department of Cardiothoracic Surgery, Affiliated Hangzhou First People's Hospital, \\ Zhejiang University School of Medicine, Hangzhou, Zhejiang 310006; ${ }^{2}$ Department of Tuberculosis, \\ Affiliated Hangzhou Chest Hospital, Zhejiang University School of Medicine; ${ }^{3}$ Department of Tuberculosis, \\ Hangzhou Red Cross Hospital, Hangzhou, Zhejiang 310003; ${ }^{4}$ Department of Cancer Diagnosis and Treatment, \\ Institute of Cancer and Basic Medicine, Chinese Academy of Sciences; ${ }^{5}$ Department of Thoracic Radiotherapy, \\ Cancer Hospital of The University of The Chinese Academy of Sciences; ${ }^{6}$ Department of Thoracic Radiotherapy, \\ Zhejiang Cancer Hospital, Hangzhou, Zhejiang 310000, P.R. China
}

Received November 5, 2020; Accepted March 11, 2021

DOI: $10.3892 / \mathrm{mmr} .2021 .12109$

\begin{abstract}
Abnormal circular RNAs (circRNAs) are associated with biological processes in cancer; however, the function of circRNAs remains largely unknown in non-small cell lung cancer (NSCLC). The present study aimed to investigate the role of hsa_circ_0058357 on the progression of NSCLC. Cell proliferation, migration and apoptosis were determined using Cell Counting Kit-8, Transwell and flow cytometry assays, respectively. Gene [circRNA and microRNA (miR)] and protein expression levels were determined via reverse transcription-quantitative PCR and immunoblotting. A luciferase assay was employed to detect the binding of miR-24-3p with AVL9 cell migration associated ( $A V L 9$ ), while a cancer xenograft model was established to evaluate cancer growth in vivo. The results demonstrated that hsa_circ_0058357 was highly expressed in human NSCLC tissues and NSCLC cells compared with para-cancerous tissues and human bronchial epithelial (HBE) cells, respectively. Knockdown of hsa_circ_0058357 significantly suppressed cell viability,migration and tumor growth, while it promoted apoptosis in NSCLC cells. As a competing endogenous RNA, hsa_circ_0058357 knockdown contributed to the increase of miR-24-3p expression in NSCLC cells. Of note, overexpression of miR-24-3p markedly abolished the exogenous hsa_circ_0058357-induced excessive proliferation, migration and apoptosis resistance of
\end{abstract}

Correspondence to: Dr Wei Feng, Department of Thoracic Radiotherapy, Zhejiang Cancer Hospital, 38 Guangji Road, Gongshu, Hangzhou, Zhejiang 310000, P.R. China

E-mail: fengweibrian66@126.com

*Contributed equally

Key words: hsa_circ_0058357, non-small cell lung cancer, microRNA-24-3p, AVL9 cell migration associated
NSCLC cells. Mechanistically, as a signaling molecule in late secretory pathway, AVL9 was also expressed at a high level in NSCLC tissues and cells, which could be directly suppressed by miR-24-3p. In the tumor tissues, along with growth inhibition, hsa_circ_0058357 knockdown also mediated the elevation of miR-24-3p and the reduction of AVL9. Thus, it was suggested that hsa_circ_0058357 may be a crucial regulation factor in NSCLC by sponging hsa-miR-24-3p, leading to a decrease in miR-24-3p expression, and subsequent increase in AVL9 expression. Therefore, hsa_circ_0058357 may serve as a potential target for diagnosis and gene therapy for NSCLC.

\section{Introduction}

As the most common type of lung cancer, non-small cell lung cancer (NSCLC) accounts for 80-85\% of all lung cancer types (1). Lung cancer was the leading cause of cancer-related mortality in the United States in 2020 (2). The activation or inactivation of signaling pathways and the physiological activities activated by multiple gene alterations, including mutations, amplifications, deletions and fusions, aggravate the disease progression of NSCLC (3). Despite significant advances in therapeutic strategies of NSCLC, early diagnosis remains a challenge, and thus, most patients are diagnosed with advanced lung cancer, and these patients exhibit poor prognosis $(4,5)$. The limited efficacy of conventional chemotherapy and tumor metastasis are the predominant challenges in the treatment of NSCLC (6), and so, the identification of ideal diagnostic biomarkers for early detection and elucidation of the mechanisms underlying NSCLC metastasis are urgently required.

Circular RNA (circRNAs), a class of non-coding RNAs (ncRNAs), can serve as competing endogenous RNAs (ceRNAs) or microRNA (miRNA/miR) sponges, resulting in miRNA degradation or translation inhibition (7). Dysregulated circRNAs in cancer binds to the mRNA 3'untranslated region (UTR) via miRNA response elements and affect the characteristics of tumor cells $(8,9)$. 
hsa_circ_0003998 upregulation accelerates the tumorigenesis of NSCLC by elevating the expression level of Notch receptor 1 via sponging miR-326 (10). On the basis of the regulatory mechanism of ceRNAs, hsa_circ_0023404 also induces tumor progression of NSCLC by regulating the miR-217/zinc finger E-box-binding homeobox 1 axis (11). Moreover, hsa_circ_0008305 inhibits TGF- $\beta$-induced epithelial-mesenchymal transition (EMT) and metastasis by regulating tripartite motif containing 24 (TRIM24) in NSCLC (12). Therefore, the regulatory mechanism of ncRNAs/miRNAs serves an important role during the progression of NSCLC. Although the role of circRNAs on the development of NSCLC has been extensively investigated, the underlying mechanisms via which circRNA mediate oncogenic pathways that are involved in NSCLC progression remain poorly understood.

AVL9 cell migration associated ( $A V L 9)$ is a crucial conserved protein in the late secretory pathway and is required for the generation of secretory vesicles, as well as for actin polarization and polarized growth (13). Depletion of AVL9 results in secretory defects and the accumulation of Golgi-like membranes (14). AVL9 is a key regulatory factor in intracellular trafficking and cell cycle progression, and AVL9 knockdown-induced abnormalities are derived from both aberrant intracellular trafficking and defective mitosis, leading to an elevated rate of cell apoptosis (15). In colorectal cancer, AVL9-mediated epithelial cell polarity control may suppress colorectal tumor progression (16). These data suggest that $A V L 9$ may be a cancer driver candidate gene. A previous study reported that LINC00662, which belongs to the class of ncRNAs with length of $>200$ nucleotides known as long ncRNAs (IncRNAs), initiates colorectal cancer progression and metastasis by competing with miR-497-5p, which subsequently represses the expression of AVL9 (17). However, whether the ncRNAs/miRNAs axis, especially circRNAs/miRNAs, affects oncogenesis of NSCLC by targeting AVL9 remains poorly understood.

Herein, the present study aimed to identify the expression pattern of hsa_circ_0058357 [produced from the autophagy related 9A (ATG9A) gene] in NSCLC. The current study further investigated the underlying regulation mechanism of hsa_circ_0058357 on the proliferation, migration, apoptosis and tumor growth of NSCLC cells via sponging miR-24-3p and regulating AVL9 expression. The present findings could highlight the role of circ_0058357 in NSCLC and provide a novel drug target for treating NSCLC.

\section{Materials and methods}

Subjects. A total of 20 fresh human NSCLC tissues and paired-paracancerous tissues located $2 \mathrm{~cm}$ away from the lesion were collected from patients with NSCLC (6 females and 14 males) at the Affiliated Hangzhou First People's Hospital (Hangzhou, China) between March 2015 and September 2018. The age of all patients ranged from 45-58 years. All samples were analyzed retrospectively in this study. All experiments were approved by the local Ethics Committee of Affiliated Hangzhou First People's Hospital (ethical approval no. 2016HZFPH-B072Z), and signed informed consent was obtained from all the patients. All fresh samples were immediately frozen in liquid nitrogen $\left(-196^{\circ} \mathrm{C}\right)$ and maintained for subsequent experimentations.

Cell culture and transfection. The human bronchial epithelial (HBE) cell line and NSCLC cell lines, including H292, A549, H1299 and H460, were obtained from the American Type Culture Collection. Cells were all cultured in complete growth medium (DMEM; Gibco; Thermo Fisher Scientific, Inc.) containing 10\% FBS (cat. no. 10099-141; Gibco; Thermo Fisher Scientific, Inc.) at $37^{\circ} \mathrm{C}$ with $5 \% \mathrm{CO}_{2}$.

For cell transfection, A549 and H1299 cells were seeded at a density of $5 \times 10^{5}$ in 6-well plates. After incubation overnight at $37^{\circ} \mathrm{C}$ with $5 \% \mathrm{CO}_{2}, 2 \mu \mathrm{l}$ circ_0058357 small interfering (si)RNA (100 mM), circ_0058357 overexpression (OE) plasmid or $50 \mu \mathrm{M}$ miR-24-3p mimic were transfected into cells for $48 \mathrm{~h}$ using Lipofectamine ${ }^{\circledR} 2000$ at room temperature (cat. no. 11668-019; Invitrogen; Thermo Fisher Scientific, Inc.), according to the manufacture's introduction. The siRNA targeting circ_0058357 and the miR-24-3p mimic were synthesized by Guangzhou RiboBio Co., Ltd. (Table I). The recombinant circ_0058357 OE plasmid was constructed using PLCDH-ciR scrambled control vector (Biovector Science Lab, Inc). Following $48 \mathrm{~h}$ of transfection, all cells were used for subsequent experiments.

Cell Counting Kit (CCK)-8 assay. After transfection with circ_0058357 siRNA or co-transfected with circ_0058357 OE plasmid and miR-24-3p mimic, A549 and H1299 cells were plated at 96 -well plate at a density of $3 \times 10^{3} /$ well. After incubation for $24,48,72$ and $96 \mathrm{~h}$ at $37^{\circ} \mathrm{C}$ with $5 \% \mathrm{CO}_{2}, 10 \mu \mathrm{lCCK}-8$ (cat. no. BS350B; Biosharp Life Sciences) was added to each well for an additional $4 \mathrm{~h}$ incubation at $37^{\circ} \mathrm{C}$, according to the manufacturer's protocol. Then, the absorption optical density value of cells was determined using a microplate reader (MULTISKAN MK3; Thermo Fisher Scientific, Inc.) at the wavelength of $450 \mathrm{~nm}$.

Flow cytometry. A549 and H1299 cells transfected with circ_0058357 siRNA or the combination of circ_0058357 OE plasmid and miR-24-3p mimic were digested using $0.25 \%$ trypsin (Gibco; Thermo Fisher Scientific, Inc.) and resuspended in $500 \mu \mathrm{l}$ Annexin $\mathrm{V}$ binding-buffer. After adding $5 \mu 1$ 7-AAD and $10 \mu 1$ PI (Sigma Aldrich; Merck KGaA), the mixture of the binding-buffer and cells was incubated for $20 \mathrm{~min}$ in darkness at room temperature. Then, the proportion of apoptotic cell was determined via flow cytometry (CytoFLEX; Beckman Coulter, Inc.) and analyzed using ModFit LT software (v3.3; Becton, Dickinson and Company). The apoptotic rate was calculated based on the percentage of early and late apoptotic cells. Each experiment was performed at least in triplicate.

Transwell and wound healing assays. Migration assays of A549 and H1299 cells were detected using Transwell chambers (cat. no. 353097; BD Biosciences). Briefly, $800 \mu \mathrm{l}$ complete medium containing $10 \%$ FBS was added into the lower chambers. Then, $200 \mu 1$ A549 and H1299 cells $\left(3 \times 10^{5}\right.$ cells $\left./ \mathrm{ml}\right)$ resuspended in DMEM were transferred to the upper chambers and allowed to migrate at $37^{\circ} \mathrm{C}$ in $5 \% \mathrm{CO}_{2}$ for $28 \mathrm{~h}$. Subsequently, cells in the upper surface of top chambers were removed using cotton swab. Cells migrating to the 
Table I. Sequences of siRNA circ_0058357.

Name Sequence

siRNA1 circ_0058357 5'-GCTCATGCAGTTCCTCTTT-3'

siRNA2 circ_0058357 5'-GGATCCACCGGCTTATCAA-3'

siRNA3 circ_0058357 5'-CCAGATCTGCATCCACAAA-3' SiRNA NC 5'-TTCTCCGAACGTGTCACGT-3'

siRNA, small interfering RNA; circ, circular RNA; NC, negative control.

bottom surface were stained for $20 \mathrm{~min}$ at room temperature with $0.5 \%$ crystal violet dye (Sigma-Aldrich; Merck KGaA). The number of migrated cells was counted using a DMR inverted microscope (magnification, $\mathrm{x} 40$; OLYMP ${ }^{\circledR} \mathrm{S}$, IX51; Olympus Corporation).

For the wound healing assay, cells were scratched to create a linear wound with the pipette tip (200- $\mu \mathrm{l})$ at the confluence of $90-95 \%$, which was followed by an incubation in serum-free RPMI-1640 medium (Gibco; Thermo Fisher Scientific, Inc.) for $24 \mathrm{~h}$. Then, cells were imaged using a light microscope (magnification, x10) and the percent of wound closure was calculated by analyzing the wound area using Image Pro Plus (v7.0; Media Cybernetics, Inc.).

Western blot analysis. Total protein from cells and tumor tissues was extracted using RIPA buffer (Beyotime Institute of Biotechnology). Protein determination was performed using a BCA kit (Beyotime Institute of Biotechnology). Equal amounts of protein $(30 \mu \mathrm{g})$ were subjected to $10 \%$ polyacrylamide gel electrophoresis for $2 \mathrm{~h}$ and then all protein was transferred to a PVDF membrane. After blocking with 5\% skimmed milk for $1 \mathrm{~h}$ at room temperature, the activated PVDF membrane was incubated with an antibody against AVL9 (cat. no. GTX16209; 1:2,000; GeneTeX, Inc.) overnight at $4^{\circ} \mathrm{C}$. GADPH (cat. no. ab181602; 1:5,000; Abcam) served as the internal control. The following day, the membrane was incubated with the HRP-conjugated secondary antibody (1:5,000; cat. no. BA1054; Boster Biological Technology) for $1 \mathrm{~h}$ at room temperature and the bands were visualized using an ECL reagent (EMD Millipore). Densitometric analysis was performed using Image Pro Plus software.

Luciferase assay. The target miRNAs of circ_0058357 were identified using the online StarBase database (http://starbase. sysu.edu.cn/index.php). Subsequently, the wild-type (WT) or mutant (MUT) 3'-UTR sequence of AVL9 and WT or MUT circ_0058357 were amplified using genomic DNA and sub-cloned into the region directly downstream of the stop codon in the luciferase reporter vector (pGL4-Basic system; Addgene, Inc.). For the analysis of the relationship between circ_0058357 and miR-24-3p, A549 and H1299 cells were co-transfected with $50 \mu \mathrm{M}$ miR-24-3p mimic and $0.5 \mu \mathrm{g}$ circ_0058357 ${ }^{\mathrm{WT}}$ or circ_0058357 ${ }^{\mathrm{MUT}}$ plasmids using Lipofectamine 2000. For the verification of the association between miR-24-3p and AVL9, the two cell lines were co-transfected with miR-24-3p mimic and AVL9 ${ }^{\mathrm{WT}}$ or AVL9 ${ }^{\mathrm{MUT}}$ plasmids. After transfection for
48 h, firefly and Renilla luciferase activities were measured using the dual-luciferase reporter assay system (Promega Corporation) and the luciferase activities were calculated based on the ration between firefly luciferase activities and Renilla luciferase activities.

Reverse transcription-quantitative $(R T-q) P C R$. Total RNA was extracted from tumor cells and tissues and purified using TRIzol ${ }^{\circledR}$ reagent (Invitrogen; Thermo Fisher Scientific, Inc.) and an RNeasy Mini kit (Qiagen, Inc.) according to the manufacturer's instructions. Then, $10 \mathrm{ng}$ RNA was reverse transcribed into cDNA using a TaqMan ${ }^{\mathrm{TM}}$ miRNA RT kit (cat. no. 4366596; Thermo Fisher Scientific, Inc.) at $25^{\circ} \mathrm{C}$ for $5 \mathrm{~min}, 50^{\circ} \mathrm{C}$ for $15 \mathrm{~min}, 85^{\circ} \mathrm{C}$ for $5 \mathrm{~min}$ and $4^{\circ} \mathrm{C}$ for $10 \mathrm{~min}$. qPCR was subsequently performed using a SYBR Green Master mix (Vazyme Biotech Co., Ltd.) on an ABI 7500 system (Applied Biosystems; Thermo Fisher Scientific, Inc.). The following thermocycling conditions were used for the qPCR: Initial denaturation at $50^{\circ} \mathrm{C}$ for $2 \mathrm{~min}$ and $95^{\circ} \mathrm{C}$ for $10 \mathrm{~min}$; followed by 40 cycles of $95^{\circ} \mathrm{C}$ for $30 \mathrm{sec}$ and $60^{\circ} \mathrm{C}$ for $30 \mathrm{sec}$. U6 and GAPDH served as the internal control. All primer information of miRNA and circRNA is shown in Table II. After the PCR reaction, the data were normalized to the selected reference gene using U6 or GAPDH. Relative gene expression levels were quantified using the $2^{-\Delta \Delta \mathrm{Cq}}$ method (18).

Xenograft model. Male nude mice (BALB/c; age, 4 weeks; weight, 18-20 g) were purchased from Charles River Laboratories, Inc., and housed in a specific-pathogen-free room under a controlled temperature $\left(20 \pm 2^{\circ} \mathrm{C}\right)$ and $45-55 \%$ humidity. All mice were exposed to a 12-h light-dark cycle with free access to standard rodent chow and water. All experiments were approved by the Animal Ethics Committee of The Affiliated Hangzhou First People's Hospital (approval no. 2016HZFPH-AB19Z). After 1 week of adjustable feeding, $\sim 0.2 \mathrm{ml}\left(1 \times 10^{7} / \mathrm{ml}\right)$ A549 cells transfected with circ_0058357 shRNA or negative control (NC) shRNA, or cells that were not transfected were inoculated subcutaneously into the right flank of mice ( $n=8 /$ each group). After 1 week of injection, tumor size was detected once a week for consecutive 6 weeks. Tumor growth rates were determined by measuring the two orthogonal dimensional diameters of each tumor. Tumor volumes were calculated according to the formula $V=1 / 2 \mathrm{xa}^{2} \mathrm{xb}$ (with $\mathrm{a}=$ short axis, and $\mathrm{b}=$ long axis). At 7 weeks post-injection, mice were sacrificed and the tumors were completely removed. After imaging and weighing, the tumor tissues were stored at $-80^{\circ} \mathrm{C}$ for the subsequent assays. Mice were euthanasia via an overdose of $\mathrm{CO}_{2}$ (30\% volume/min) in the following situations: Weight loss of $>15 \%$ of mouse body weight, or mice continued to suffer pain from the tumor burden, such as the tumor diameter reaching to $>2.5 \mathrm{~cm}$ and ulcerated tumors. In this research, the long and short diameters of the largest tumor were as follows: Long diameter, $1.55 \mathrm{~cm}$; and short diameter, $1.12 \mathrm{~cm}$.

Immunohistochemistry (IHC). Tumor tissues were fixed with $4 \%$ paraformaldehyde for $>24 \mathrm{~h}$ at $4^{\circ} \mathrm{C}$ and embedded in paraffin. For IHC assay, after heating in an oven for $30 \mathrm{~min}$ at $60^{\circ} \mathrm{C}, 5-\mu \mathrm{m}$ thick slides of tumor tissues were deparaffinized in xylene and rehydrated in gradient ethanol, then retrieved 
Table II. Primer sequences for reverse transcription-quantitative PCR.

\begin{tabular}{lll}
\hline Name & Primer & \multicolumn{1}{c}{ Sequence } \\
\hline GAPDH & Forward & 5'-TCAAGAAGGTGGTGAAGCAGG-3' \\
& Reverse & 5'-TCAAAGGTGGAGGAGTGGGT-3' \\
circ_0058357 & Forward & 5'-GCACAGGGCTGAAGTCGC-3' \\
& Reverse & 5'-GACCTTGACGGGTTCAGTAGG-3' \\
U6 & Forward & 5'-CGCTTCGGCAGCACATATAC-3' \\
& Reverse & 5'-AAATATGGAACGCTTCACGA-3' \\
hsa-miR-24-3p & Forward & 5'-TGCGCGAGTTCAGCAGGAACAG-3' \\
& Loop & 5'-GTCGTATCCAGTGCAGGGTCCGAGGTATTCGCACTGGATACGACCTGTTCCT-3'
\end{tabular}

circ, circular RNA; miR, microRNA.
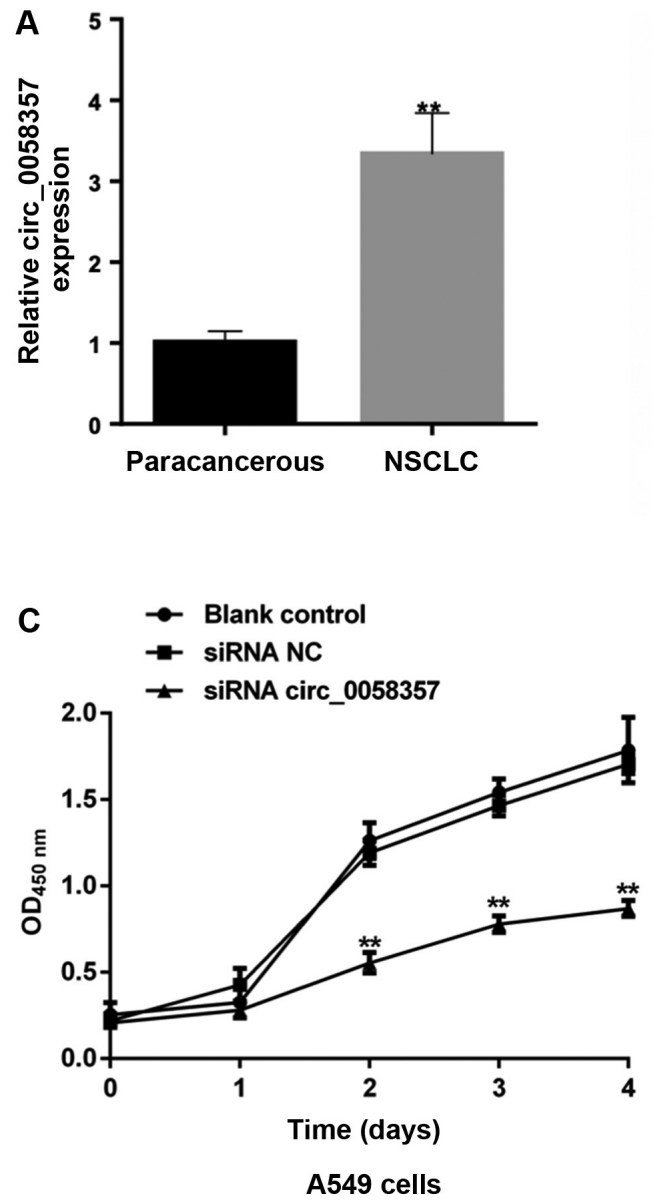
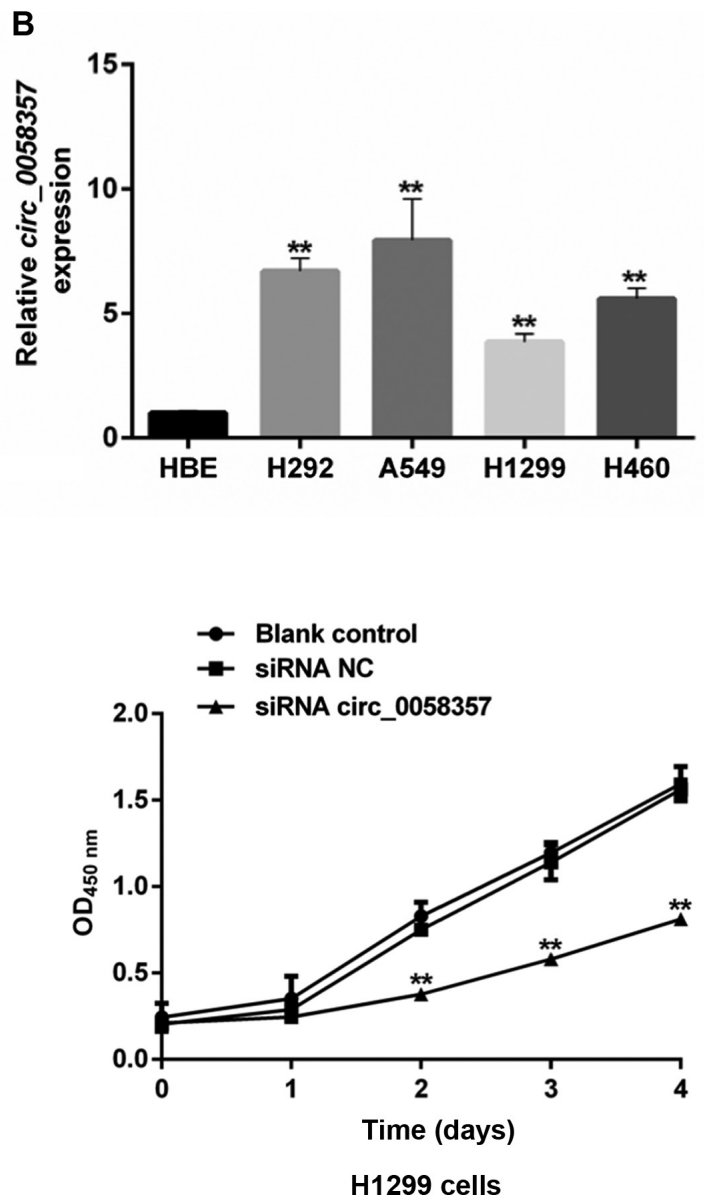

Figure 1. Effects of circ_0058357 on the viability of NSCLC cells. (A) Relative expression level of circ_0058357 in human NSCLC tissues and paired-paracancerous tissues as determined by RT-qPCR, ( $n=20 /$ group). ${ }^{* *} \mathrm{P}<0.01$ vs. paracancerous. (B) Relative expression level of circ_0058357 in NSCLC cells, including H292, A549, H1299, H460 and HBE cells as detected by RT-qPCR. ${ }^{* *}$ P<0.01 vs. HBE cells. (C) A549 and H1299 cells were transfected with siRNA NC and circ_0058357 siRNA. Then, cell viability was measured using a Cell Counting Kit-8 assay at days $1,2,3$ and 4 after incubation. ${ }^{* *} \mathrm{P}<0.01$ vs. A549 cells/H1299 cells transfected with siRNA NC. HBE, human bronchial epithelial; RT-qPCR, reverse transcription-quantitative PCR; NC, negative control; siRNA, small interfering RNA; OD, optical density; circ, circular RNA; NSCLC, non-small cell lung cancer.

by $0.01 \mathrm{M}$ citrate salt solution ( $\mathrm{pH} 6.0)$ using a microwave method. Then, endogenous peroxidase was blocked at room temperature using $3 \% \mathrm{H}_{2} \mathrm{O}_{2}$ for $15 \mathrm{~min}$. After blocking with 5\% BSA (Beijing Solarbio Science \& Technology Co., Ltd.) for $1 \mathrm{~h}$ at room temperature, a primary antibody against Ki67 (1:200; cat. no. ab15580; Abcam) was incubated with slides at $4^{\circ} \mathrm{C}$ overnight and the slides were then washed thrice with PBS-0.05\% Tween 20. Subsequently, a secondary antibody (1:500; cat. no. PV-9001; OriGene Technologies, Inc.) was incubated with slides for an additional $15 \mathrm{~min}$ at room temperature. Antigen-antibody complexes were visualized using DAB staining buffer (cat. no. ZLI-9017; OriGene 
A

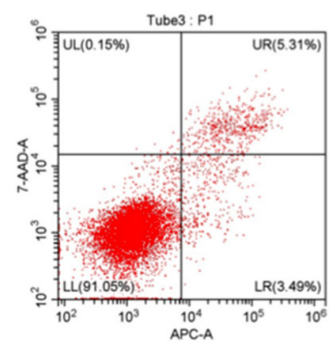

Blank control

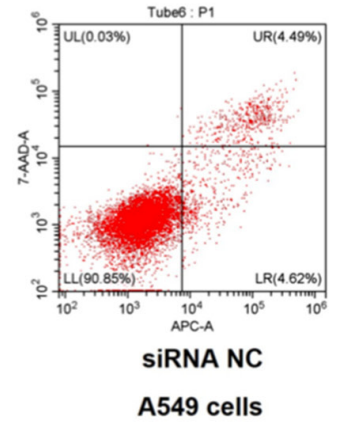

A549 cells

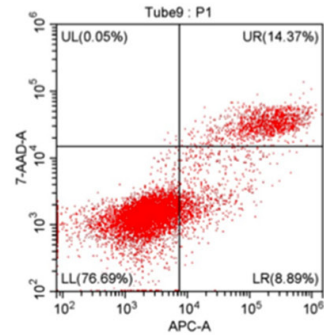

siRNA circ_0058357

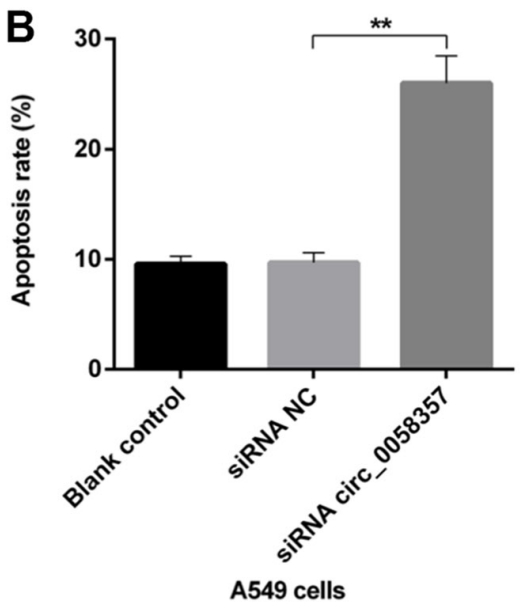

C

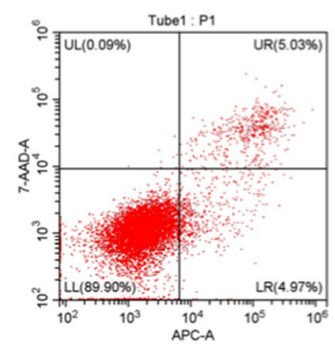

Blank control

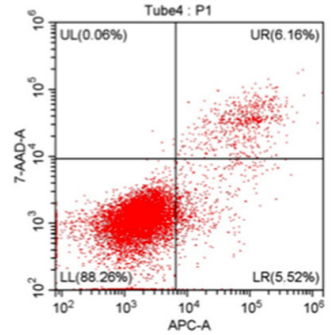

SIRNA NC

H1299 cells

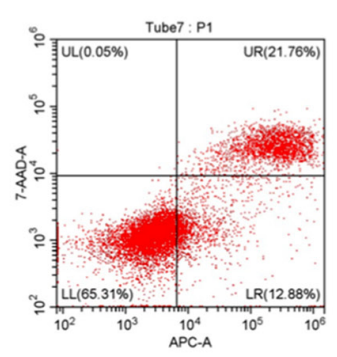

siRNA circ_0058357

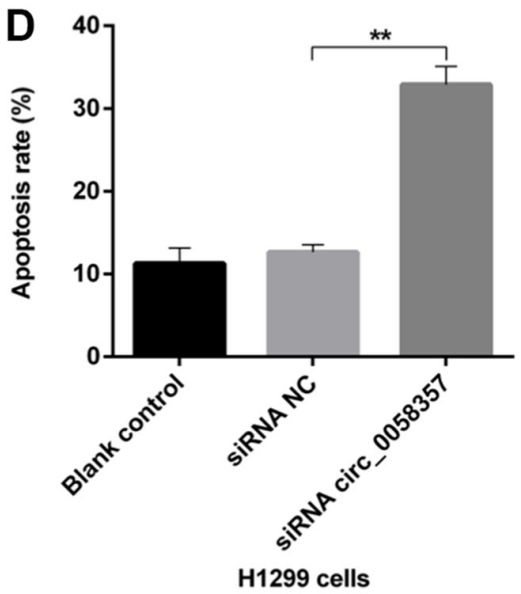

Figure 2. Effects of circ_0058357 on the apoptosis of non-small cell lung cancer cells. (A) A549 cells were transfected with siRNA NC and circ_0058357 siRNA. The proportion of apoptotic A549 cells was measured via flow cytometry. (B) Quantitative analysis of apoptotic A549 cells. (C) H1299 cells were transfected with siRNA NC and circ_0058357 siRNA. The population of apoptotic H1299 cells was determined via flow cytometry. (D) Quantitative analysis of apoptotic H1299 cells. ${ }^{* *} \mathrm{P}<0.01$. NC, negative control; siRNA, small interfering RNA; circ, circular RNA.

Technologies, Inc.). The image was observed under a biological inverted microscope (magnification, x20; IX51; Olympus Corporation).

Statistical analysis. All independent experiments were performed at least in triplicate. Data are presented as the mean \pm SD. The data for the luciferase assay results were examined using an unpaired t-test. A paired Student's t-test was employed to compare the difference between two groups. Moreover, a mixed two-way ANOVA and a Bonferroni or Sidak post hoc test was used for analysis where applicable. One-way ANOVA with Bonferroni test was conducted to analyze the differences among multiple groups ( $>2$ groups) using GraphPad Prism 6.0 (GraphPad Software, Inc.) software. $\mathrm{P}<0.05$ was considered to indicate a statistically significant difference.

\section{Results}

Knockdown of circ_0058357 inhibits the viability of NSCLC cells. To investigate the role of circ_0058357 on NSCLC, its expression pattern was firstly determined in human NSCLC tissues and NSCLC cell lines. The relative expression level of hsa_circ_0058357 was significantly elevated by $\sim 3.5$-fold in human NSCLC tissues compared with that in the paired-paracancerous tissues (Fig. 1A). Moreover, in comparison with normal HBE cells, hsa_circ_0058357 expression was upregulated in the NSCLC cell lines, such as H292, A549, H1299 and H460, with the highest level in A549 cells and a moderate increase in H1299 cells (Fig. 1B). Thus, A549 and H1299 cell lines were selected to conduct the subsequent experiments.

Given the high expression level of circ_0058357, it was suggested that it could be a tumor suppressor in NSCLC. First, the specific siRNAs targeting circ_0058357 were screened. Among the three candidate siRNAs, it was discovered that circ_0058357 siRNA-2 had the strongest inhibition efficiency on the expression of circ_0058357. Thus, circ_0058357 siRNA-2 was selected to perform the following experiments (Fig. S1).

When knocking down circ_0058357 using specific siRNA, the viability of both A549 and H1299 cells was significantly repressed compared with that in cells transfected with $\mathrm{NC}$ siRNA from days 2 to 4 (Fig. 1C). However, there was no difference in cell viability between the blank and NC siRNA groups. Therefore, the present data indicated that circ_0058357 was highly expressed in NSCLC tissues and NSCLC cells. Moreover, the knockdown of circ_0058357 contributed to the proliferation inhibition of NSCLC cells. 
A

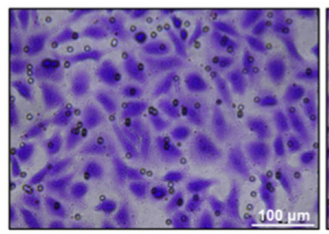

Blank control

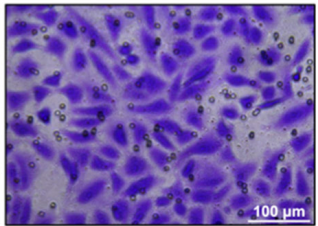

SiRNA NC

A549 cells

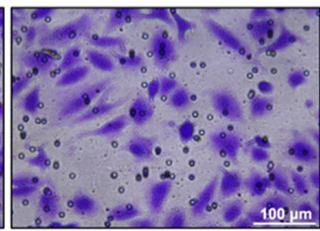

siRNA circ_0058357

B

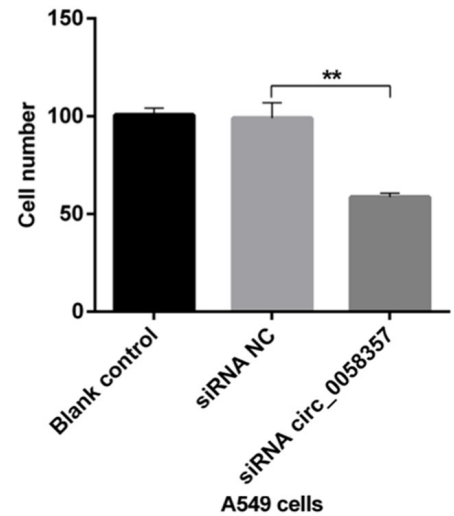

C

o h

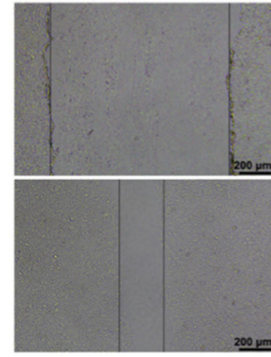

Blank control

E

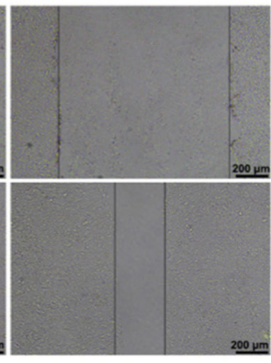

SiRNA NC

A549 cells

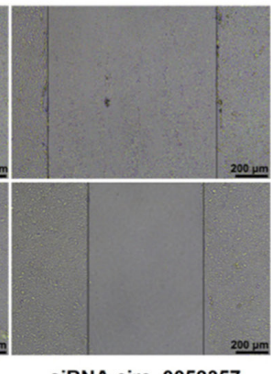

siRNA circ_0058357

D

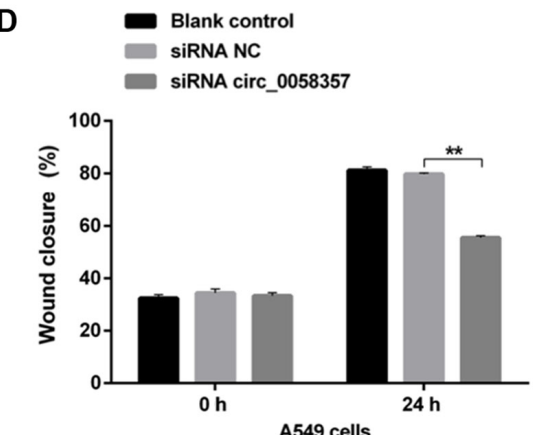

F

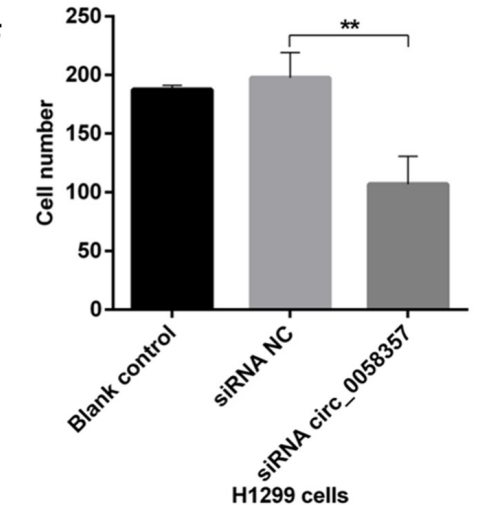

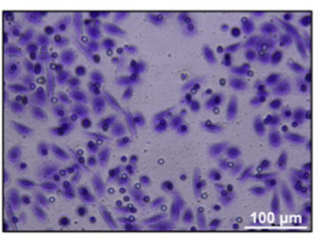

Blank control
G

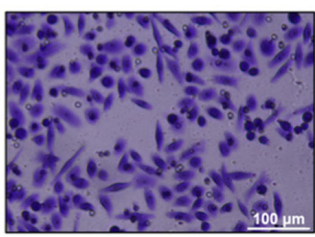

SiRNA NC

H1299 cells

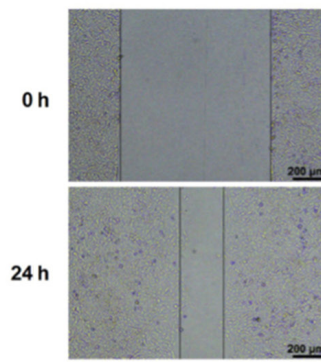

Blank control

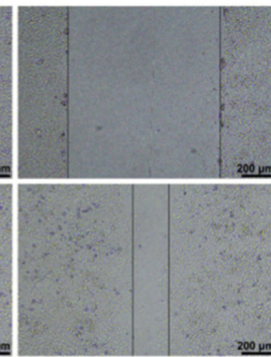

SIRNA NC

H1299 cells

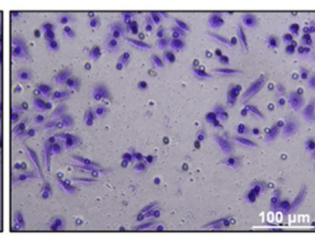

siRNA circ_0058357

H1299 cells

H
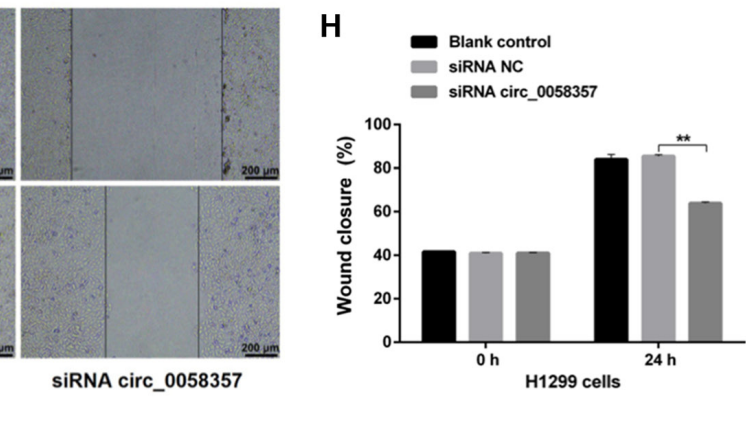

Figure 3. Effects of circ_0058357 on the migration of NSCLC cells. (A) A549 cells were transfected with siRNA NC and circ_0058357 siRNA. The migrated A549 cells were stained with crystal violet. Scale bar, $100 \mu \mathrm{m}$. (B) Quantitative analysis of migration cells in panel A. (C) After transfection with siRNA NC and circ_0058357 siRNA, A549 cells were wounded using a pipette tip. Subsequently, the cells were imaged using a microscope. Scale bar, $200 \mu \mathrm{m}$. (D) The healing rate was measured as the relative percent of wound closure. (E) H1299 cells were transfected with siRNA NC and circ_0058357 siRNA. The number of migrated H1299 cells was determined via crystal violet staining. Scale bar, $100 \mu \mathrm{m}$. (F) Quantitative analysis of migrated cells in panel E. (G) After transfection with siRNA NC and circ_0058357 siRNA, H1299 cells were wounded using a pipette tip. Subsequently, the cells were imaged using a microscope. Scale bar, $200 \mu \mathrm{m}$. (H) The healing rate was measured as the relative percent of wound closure. ${ }^{* *} \mathrm{P}<0.01$. NC, negative control; siRNA, small interfering RNA; circ, circular RNA.

Knockdown of circ_0058357 accelerates the apoptosis of NSCLC cells. Next, it was determined whether the circ_0058357 knockdown-mediated decrease of cell viability was associated with programmed cell death in NSCLC cells. Compared with the blank control A549 cells, the proportion of apoptotic cells, including early and late apoptotic cells, 
A

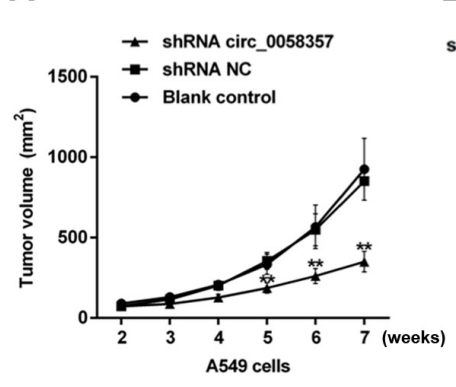

B

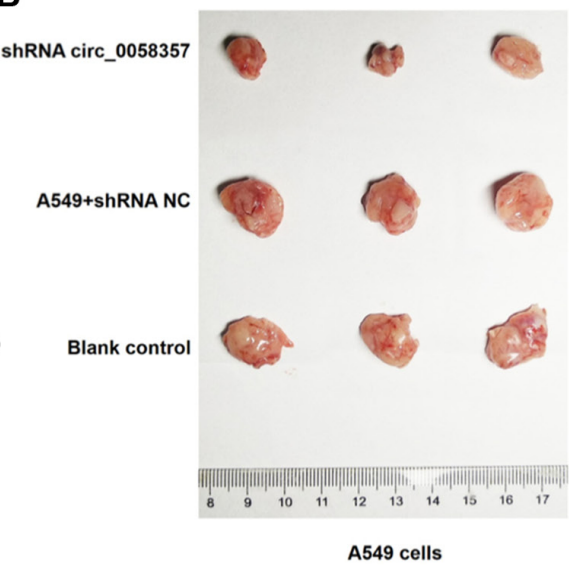

C

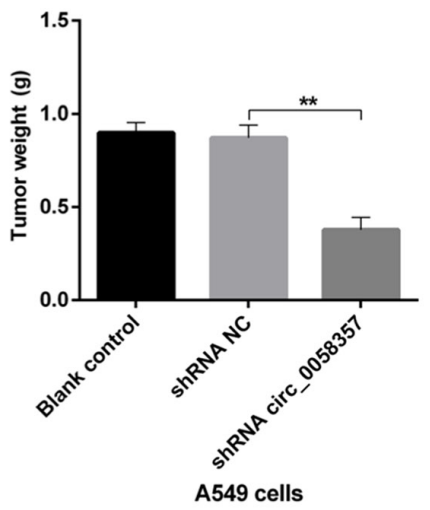

D

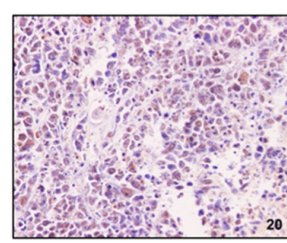

Blank control

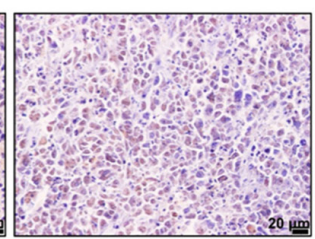

ShRNA NC

A549 cells

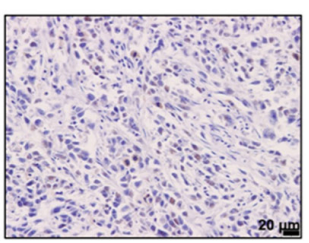

ShRNA circ_0058357

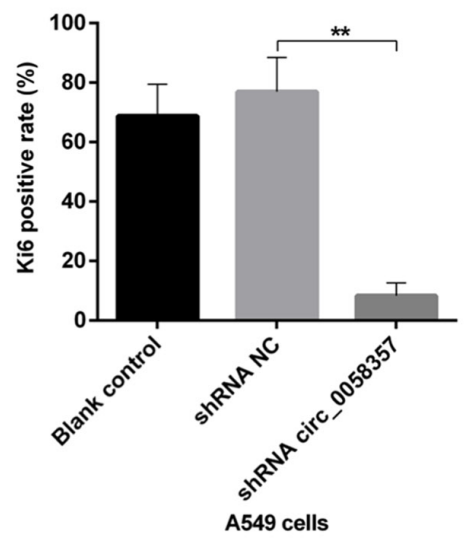

Figure 4. Role of circ_0058357 knockdown on tumor growth of NSCLC cells in vivo. (A) A549 cells were transfected with shRNA NC and circ_0058357 shRNA. Tumor size was detected once a week for consecutive 6 weeks. Then, a tumor growth curve was generated according to the tumor volume at different time points. (B) After injection for 7 weeks, tumors in different group were removed and imaged. (C) Quantitative analysis of tumor weight in the three groups, (n=8/each group). (D) Ki67 expression in tumor tissues was determined via immunohistochemistry staining. (D) The positive rate of Ki67 in tumor tissues was assessed using Image Pro Plus software. ${ }^{* *} \mathrm{P}<0.01$. NC, negative control; shRNA, short hairpin RNA; circ, circular RNA.

was not affected by the transfection of siRNA NC, while there was a $15 \%$ increase in the number of apoptotic cells in circ_0058357-siRNA group compared with the siRNA NC group (Fig. 2A and B). Consistently, in H1299 cells, knockdown of circ_0058357 also enhanced the population of apoptotic cells by $\sim 20 \%$ when compared with cells transfected with siRNA NC. Furthermore, exogenous siRNA NC had no effect on apoptotic events in untransfected H1299 cells (Fig. 2C and D). Thus, circ_0058357 knockdown-induced proliferation inhibition may be associated with the elevation of programmed cell death.

circ_0058357 knockdown impedes the migration of NSCLC cells. Then, the effect of circ_0058357 knockdown on the metastasis of NSCLC cells was determined. Based on the Transwell assay results, a $40 \%$ reduction in migrated cells was observed in A549 cells transfected with circ_0058357-siRNA when compared with the siRNA NC group (Fig. 3A and B). In the wound healing assay, circ_0058357-siRNA transfection significantly inhibited healing rate of A549 cells compared with cells transfected with siRNA NC (Fig. 3C and D). circ_0058357 knockdown also significantly limited the migratory ability of H1299 cells, showing a 50\% decrease compared with the
siRNA NC group (Fig. 3E and F). Consistently, circ_0058357 knockdown decreased the wound healing rate of H1299 cells after $24 \mathrm{~h}$ of transfection compared with the siRNA NC group (Fig. 3G and H). In both cell lines, the addition of siRNA NC did not affect the cellular migration (Fig. 3A-H). Collectively, circ_0058357 was suggested to be a key regulator during the metastasis of NSCLC cells.

circ_0058357 knockdown restrains tumor growth of NSCLC cells in vivo. Based on the tumor volumes at different time point, it was identified that circ_0058357 shRNA transfection slowed down the tumor growth rate, which presented a significant difference compared with the shRNA NC group, starting 5 weeks after injection (Fig. 4A). Additionally, knockdown of circ_0058357 in A549 cells using shRNA significantly inhibited tumor size and tumor weight by $\sim 50 \%$ at the end of tumor xenograft assay (Fig. 4B and C). Moreover, the shRNA NC did not affect the tumor growth and tumor volume compared with the blank control group. It was also found that both knockdown of circ_0058357 and the shRNA NC in A549 cells did not impact the body weight of the three groups (Fig. S2).

Next, the proliferative activity of tumor tissues in different groups was assessed using an IHC staining assay with an 
A

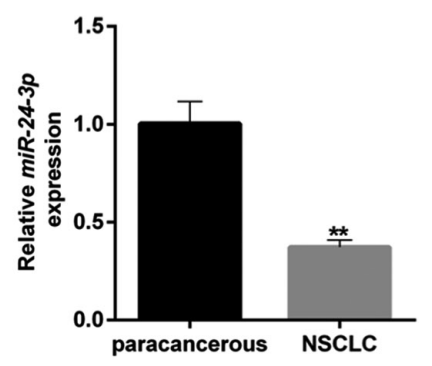

D

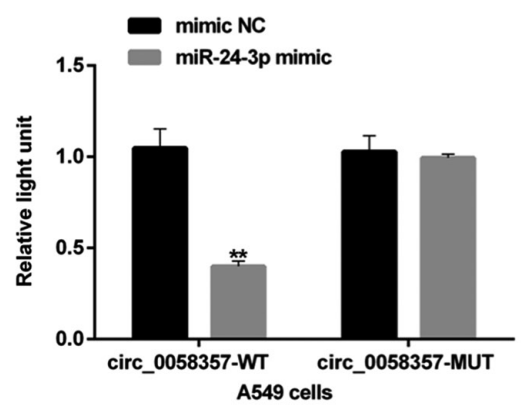

G

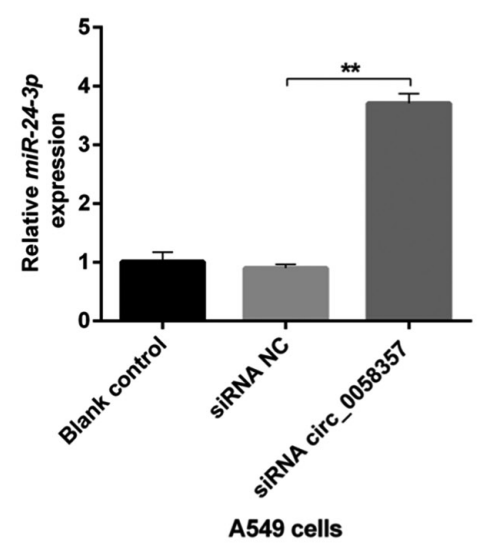

B

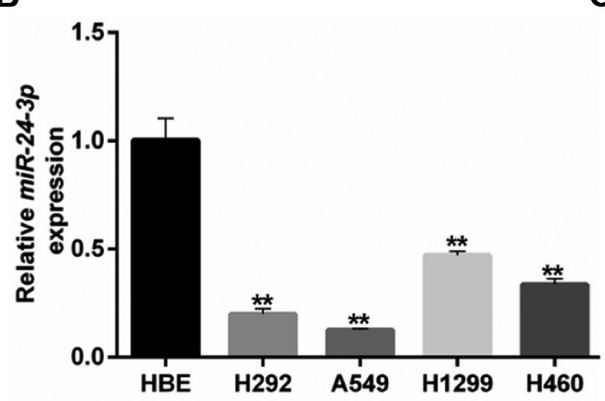

E

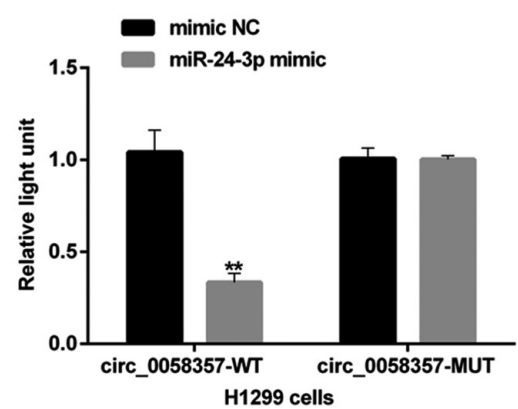

H

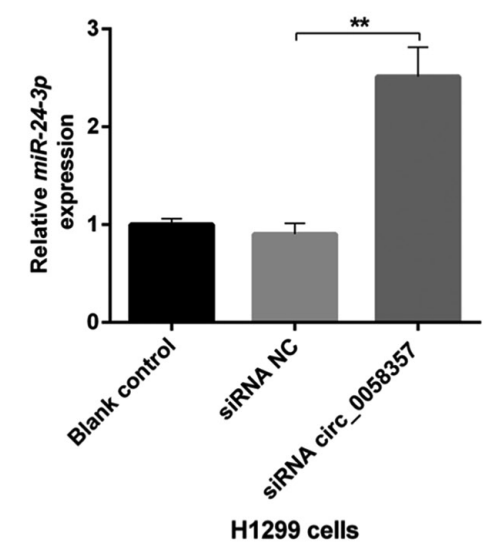

C 흠

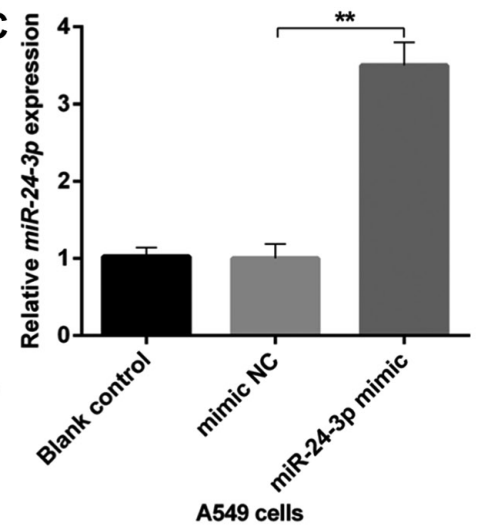

F

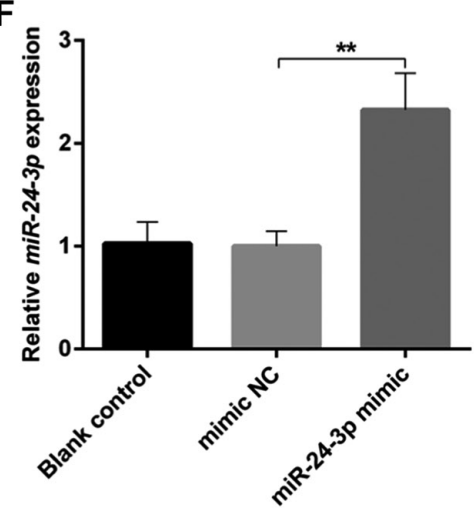

H1299 cells

Figure 5. An association between circ_0058357 and miR-24-3p in NSCLC cells. (A) Relative expression level of miR-24-3p in human NSCLC tissues and paired-paracancerous tissues, as determined via RT-qPCR, (n=20/group). (B) Relative expression level of miR-24-3p in NSCLC cells, including H292, A549, H1299 and H460, and HBE cells, as detected via RT-qPCR. (C) Transfection efficiency of miR-24-3p mimics, as determined via RT-qPCR in A549 cells. (D) A549 and (E) H1299 cells were co-transfected with miR-24-3p mimic and WT or MUT-circ 0058357, then luciferase activity was detected and normalized with Renilla. (F) Transfection efficiency of miR-24-3p mimics, as determined via RT-qPCR in H1299 cells. (G) A549 and (H) H1299 cells were firstly transfected with circ_0058357 siRNA, then the relative expression of miR-24-3p was determined via RT-qPCR. ${ }^{* *} \mathrm{P}<0.01$. NC, negative control; siRNA, small interfering RNA; circ, circular RNA; HBE, human bronchial epithelial; RT-qPCR, reverse transcription-quantitative PCR; WT, wild-type; MUT, mutant; miR, microRNA.

antibody against Ki67. A low expression level of Ki67 was observed in tumor tissues from circ_0058357-shRNA group compared with that in the shRNA NC group. Furthermore, Ki67-positive cells in circ_0058357-shRNA group accounted for only $14 \%$ of the Ki67-positive cells in tissues of shRNA NC group (Fig. 4D). Therefore, circ_0058357 knockdown facilitated the tumor growth inhibition of NSCLC cell in vivo.

hsa-miR-24-3p is sponged by circ_0058357 in NSCLC cells. Using the online StarBase database, the potential downstream sponges of circ_0058357 were identified. Among the screened
miRNAs, the alignScore 26 of miR-24-3p was ranked in the top five, and autophagy events were associated with miR-24-3p and circ_0058357 (Table SI). Therefore, miR-24-3p was selected to predict the regulatory mechanism of circ_0058357 on NSCLC.

A recent study revealed that hsa-miR-24-3p was lowly expressed in lung adenocarcinomas (19). The present study also found that miR-24-3p was lowly expressed in NSCLC tissues and NSCLC cells compared with paracancerous tissues and HBE cells, respectively (Fig. 5A and B). Interestingly, it was predicted that circ_0058357 may be a ceRNA of miR-24-3p by directly binding to the 'GACTCGGT' element 
A

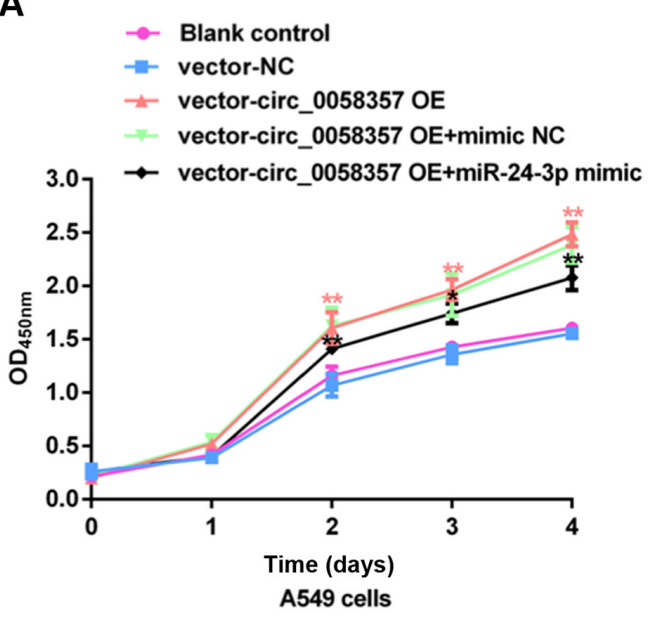

C
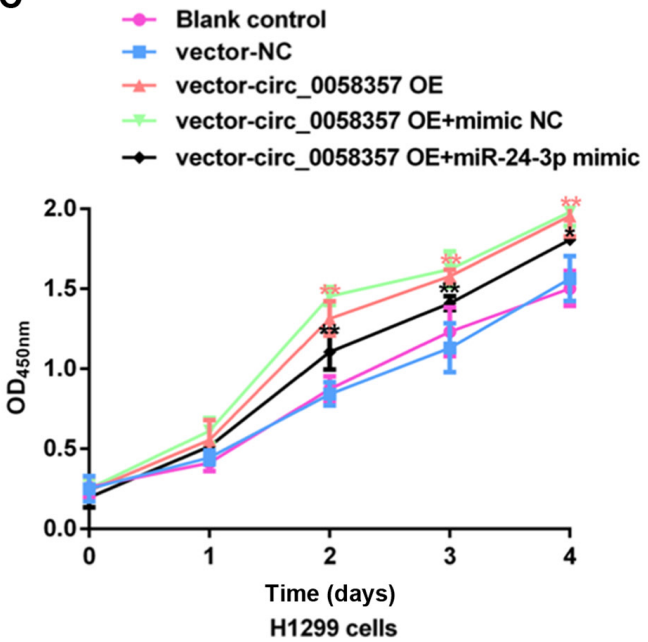

B

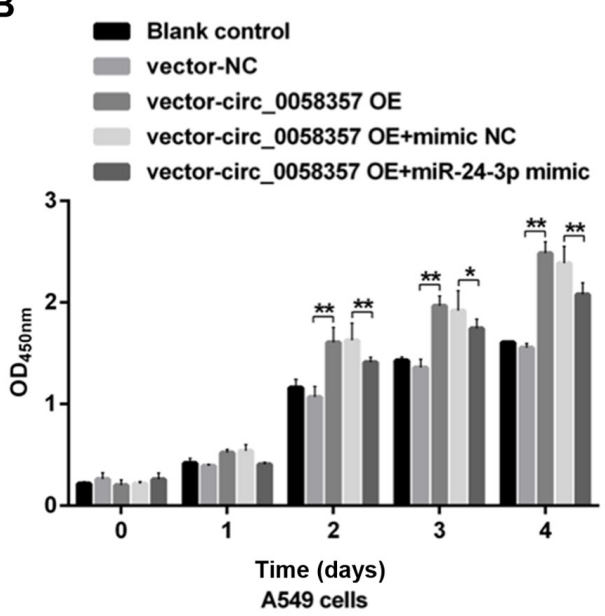

D

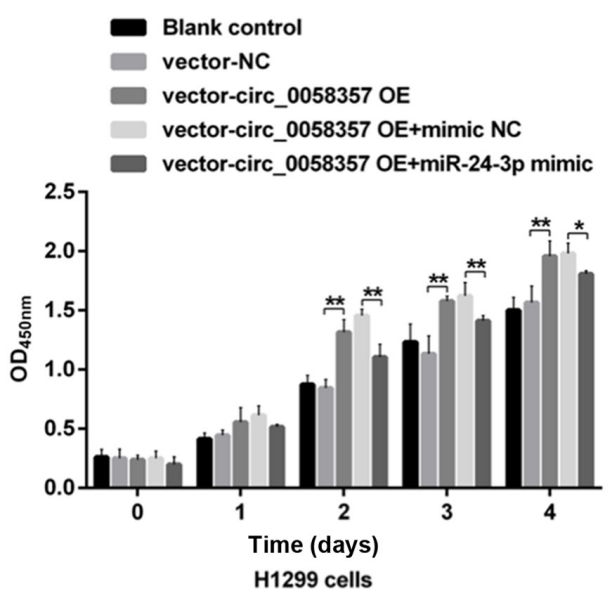

Figure 6. Effects of miR-24-3p on the exogenous circ_0058357-mediated viability of NSCLC cells. A549 and H1299 cells were transfected with vector and circ_0058357 OE plasmids alone, or in combination with vector or circ_0058357 OE plasmids and NC mimic or miR-24-3p mimic. Then, the (A) cell growth curve of and (B) viability of A549 cells, and (C) growth curve and (D) viability of H1299 cells were determined using Cell Counting Kit-8 assay at days $1,2,3$ and 4 after incubation. $\mathrm{P}<0.05,{ }^{* *} \mathrm{P}<0.01$. NC, negative control; siRNA, small interfering RNA; circ, circular RNA; OE, overexpression; OD, optical density; miR, microRNA.

of miR-24-3p (Fig. S3A). Next, A549 cells were transfected with the miR-24-3p mimic, which significantly upregulated the expression of miR-24-3p compared with mimic NC-transfected cells (Fig. 5C). The luciferase results indicated that miR-24-3p mimic transfection significantly suppressed the luciferase activity of WT circ_0058357 both in A549 and H1299 cells, while the upregulation of miR-24-3p did not affect the transcriptional activity of MUT circ_0058357 (Fig. 5D and E). It was also found that knockdown of circ_0058357 promoted the expression level of miR-24-3p, but miR-24-3p OE had no effect on the expression level of circ_0058357 in both cells (Figs. 5F-H and S3B and C). These data indicated that circ_0058357 can downregulate the expression level of miR-24-3p by directly sponging it in NSCLC cells.

miR-24-3p mimic abolishes the exogenous circ_0058357mediated proliferation promotion of NSCLC cells. Since knockdown of circ_0058357 inhibited tumorigenesis of NSCLC cells, it was suggested that OE of circ_0058357 could enhanced the oncogenesis of A549 and H1299 cells. The promotion efficiency of circ_0058357 OE plasmid was confirmed (Fig. S4). It was identified that the cell growth curve was accelerated and cell proliferation was significantly enhanced from days 2 to 4 in A549 cells transfected with circ_0058357 OE plasmid compared with cells transfected with vector (Fig. 6A and B). Moreover, exogenous circ_0058357 induced proliferation in H1299 cells after incubation for 2, 3 and 4 days (Fig. 6C and D). However, when co-transfection with circ_0058357 OE and miR-24-3p mimic, the exogenous circ_0058357-mediated promotion in cell proliferation was notably rescued in both A549 and H1299 cells (Fig. 6A-D). Thus, miR-24-3p may act as a negative regulator during circ_0058357-evoked tumorigenesis in NSCLC.

miR-24-3p mimic abrogates circ_0058357 OE-induced apoptosis inhibition and migration promotion. To further determine the regulatory role of miR-24-3p on circ_0058357-initiated biological behaviors of NSCLC cells, flow cytometry and Transwell assays were employed in cells co-transfected with 
A

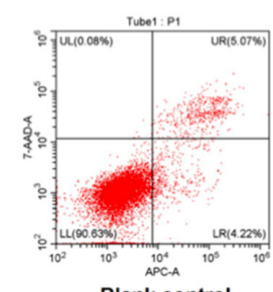

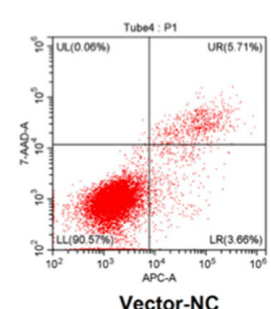

Vector-NC

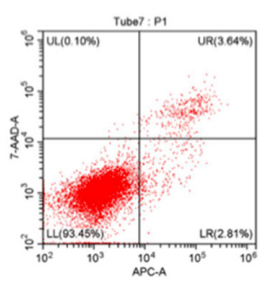

Vector-circ_0058357 OE

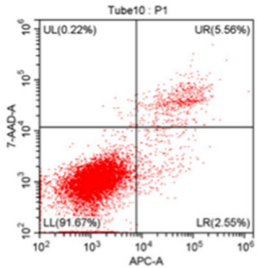

Vector-circ_0058357 OE + mimic NC

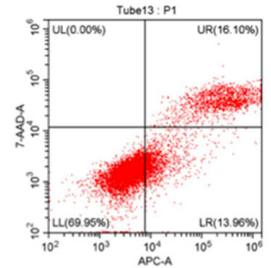

Vector-circ_0058357 OE +miR-24-3p mimic

A549 cells

C

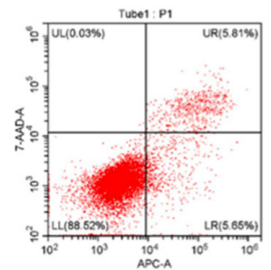

Blank control

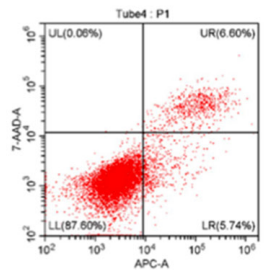

Vector-NC

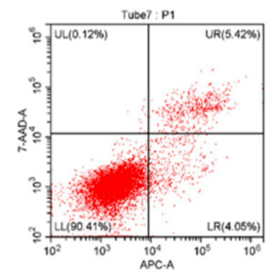

Vector-circ_0058357 OE
B

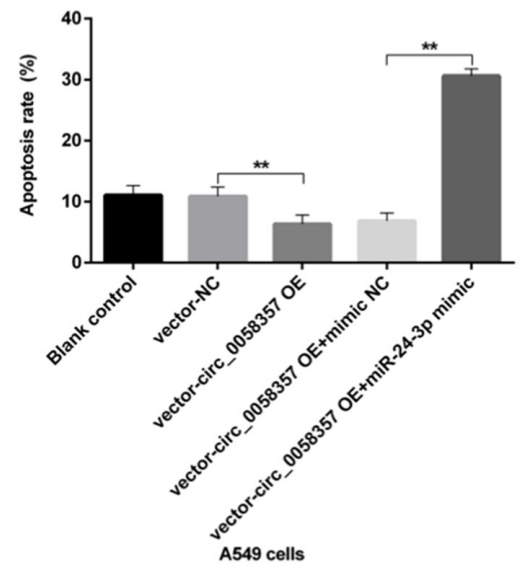

D

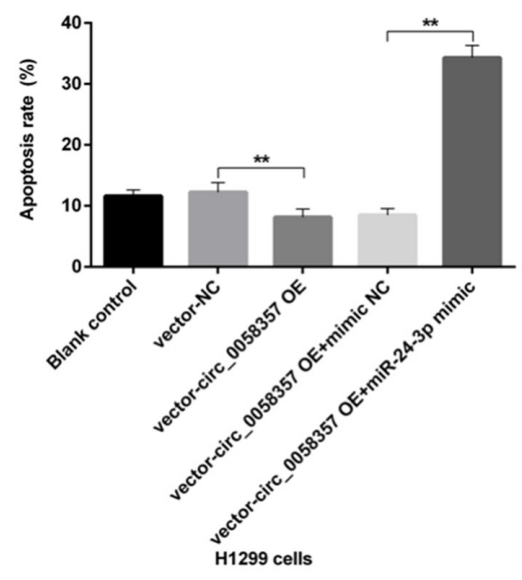

H1299 cells

Figure 7. Regulation of miR-24-3p mimic on the exogenous circ_0058357-initiated apoptosis of NSCLC cells. A549 and H1299 cells were transfected with vector and circ_0058357 OE plasmids alone, or in combination with vector or circ_0058357 OE plasmids and mimic NC or miR-24-3p mimic. (A) Proportion of apoptotic A549 cells was measured via flow cytometry. (B) Quantitative analysis of apoptotic cells in panel A. (C) The population of apoptotic H1299 cells was determined via flow cytometry. (D) Quantitative analysis of apoptotic cells in panel C. ${ }^{* *} \mathrm{P}<0.01$. NC, negative control; siRNA, small interfering RNA; circ, circular RNA; OE, overexpression; miR, microRNA.

exogenous circ_0058357 and miR-24-3p. The results demonstrated that OE of circ_0058357 repressed the number of apoptotic cells by 50\% in A549 and H1299 cells (Fig. 7A-D). However, the co-administration of miR-24-3p mimic not only abolished the inhibition role of exogenous circ_0058357, but also significantly amplified the proportion of apoptotic cells in both cell lines, showing a 5-fold increase compared with cells co-transfected with circ_0058357 OE and NC mimic, and a 3 -fold increase compared with the blank cells or cells transfected with vector-NC (Fig. 7A-D).

In the Transwell assay, after the OE of circ_0058357, there was a 1.5-fold elevation in the number of migrated A549 and H1299 cells compared with the vector-NC group (Fig. 8A-D). In comparison with the group of cells co-transfected with circ_0058357 OE and NC mimic, the miR-24-3p mimic significantly reversed the increase of cell metastasis induced by circ_0058357 OE in both cells, and returned to a normal level (Fig. 8A-D). Collectively, it was indicated that miR-24-3p negatively regulated circ_0058357-induced cell migration or the inhibition in apoptotic events.

miR-24-3p can inhibit AVL9 expression by directly binding its' 3'-UTR domain. As well as being a crucial protein in the late secretory pathway, $A V L 9$ is also considered as a cancer driver candidate gene (16). Herein, it was found that the expression level of AVL9 protein was higher in the tumor tissues from patients with NSCLC compared with that in the paired-paracancerous tissues (Fig. 9A and B). In comparison with HBE cells, there was a high expression level of AVL9 protein in NSCLC cells (Fig. 9C). 
A

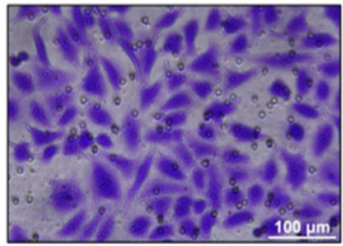

Blank control

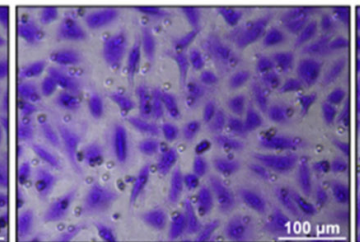

Vector-NC

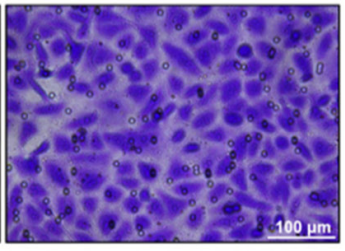

Vector-circ_0058357 OE

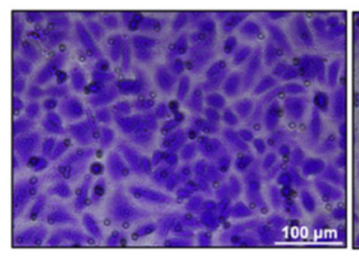

Vector-circ_0058357 $\mathrm{OE}+$ mimic NC

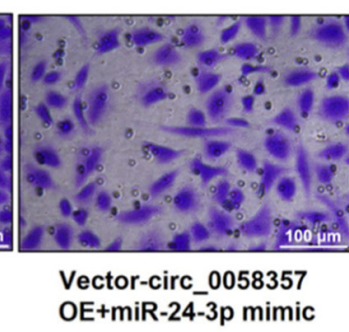

A549 cells
B

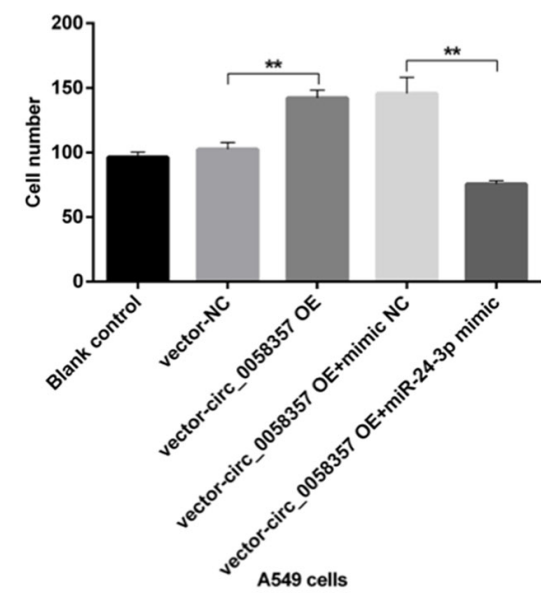

D
C

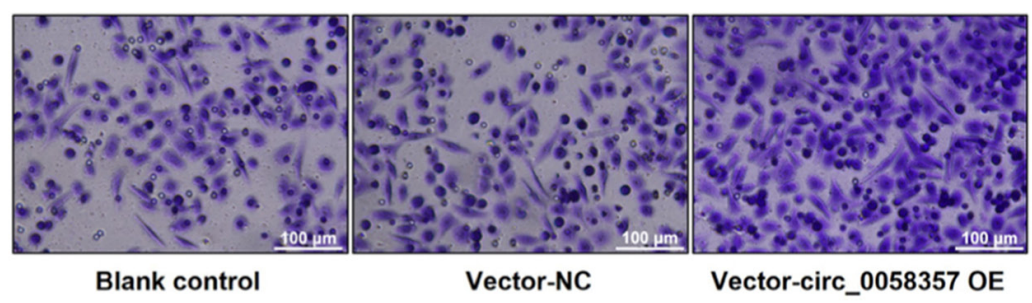

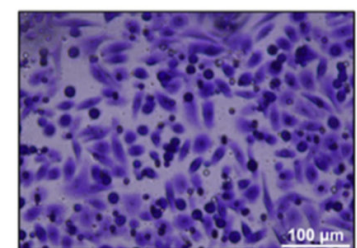

Vector-circ_0058357 $\mathrm{OE}+$ mimic $\mathrm{NC}$

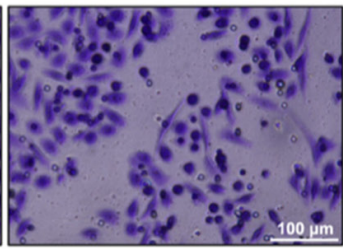

Vector-circ_0058357 OE+miR-24-3p mimic
H1299 cells

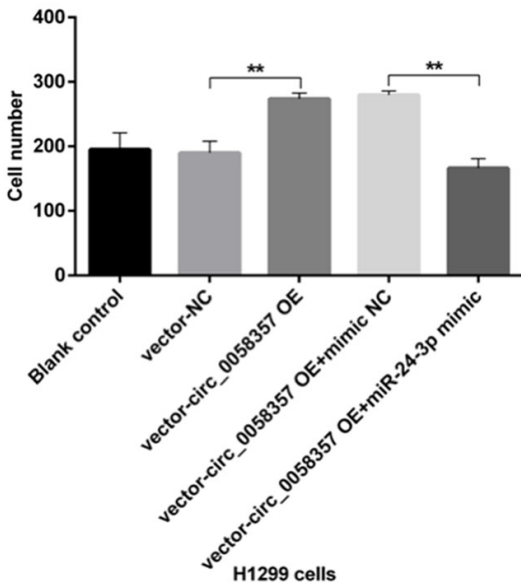

Figure 8. Effects of circ_0058357 on the migration of NSCLC cells. A549 and H1299 cells were transfected with vector and circ_0058357 OE plasmids alone or combination with transfection of vector/circ_0058357 OE plasmids and mimic NC/miR-24-3p. (A) The migration of A549 cells was detected using Transwell assay and crystal violet staining. Scale bar, $100 \mu \mathrm{m}$. (B) Quantitative analysis of migration cells in panel A. (C) The number of migrated H1299 cells in the five groups was determined via crystal violet staining. Scale bar, $100 \mu \mathrm{m}$. (D) Quantitative analysis of apoptotic cells in panel C. ${ }^{* *} \mathrm{P}<0.01$. NC, negative control; siRNA, small interfering RNA; circ, circular RNA; OE, overexpression; miR, microRNA.

Using the prediction analysis from the online StarBase database, it was observed that there was direct binding site of miR-24-3p in the $A V L 9$ gene (Fig. 9D). Utilizing the luciferase assay, the binding of miR-24-3p to AVL9 was also confirmed in both A549 and H1299 cells. The miR-24-3p mimic significantly reduced the transcriptional activity of AVL9 in the WT 3'-UTR of AVL9 group, while there was no effect on the luciferase activity in cells administrated with MUT 3'-UTR of AVL9 (Fig. 9D). Of note, transfection with exogenous miR-24-3p markedly decreased the protein expression level of AVL9 in NSCLC cells (Fig. 9E). These findings suggested that AVL9 was directly inhibited by miR-24-3p in NSCLC cells, and that AVL9 may be the key switch in the circ_0058357/miR-24-3p axis during the development of NSCLC.
A circ_0058357/miR-24-3p/AVL9 signaling axis is involved in tumor development of NSCLC in vivo. Next, the relationship between circ_0058357/miR-24-3p axis and AVL9 was assessed in the tumor tissues from xenograft model. Tumors from mice bearing A549 cells transfected with circ_0058357 shRNA had a significant decline in the expression level of circ_0058357 compared with the shRNA NC group (Fig. 10A). On the contrary, compared with the shRNA NC, miR-24-3p expression was significantly elevated by $\sim 4$-fold in tumor tissues when circ_0058357 was knocked down (Fig. 10B). Additionally, the protein expression level of AVL9 was significantly decreased in tumors of circ_0058357 shRNA group (Fig. 10C). It was also found that transfection with shRNA NC of circ_0058357 in A549 cells did not affect the circ_0058357/miR-24-3p/AVL9 
A
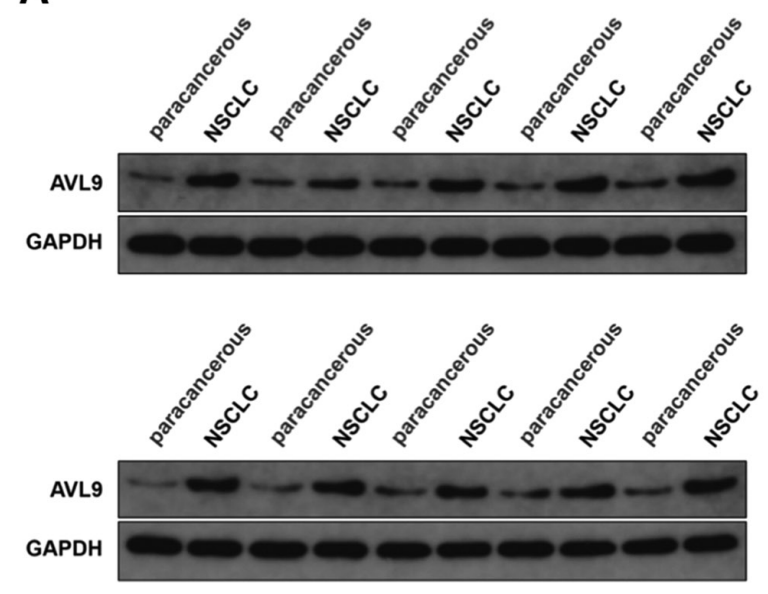

C

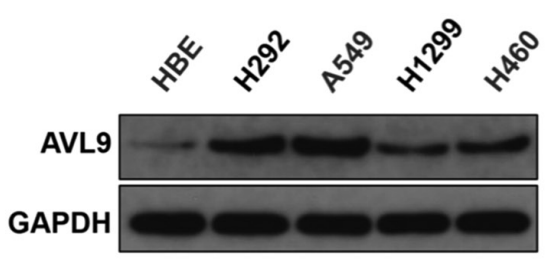

E

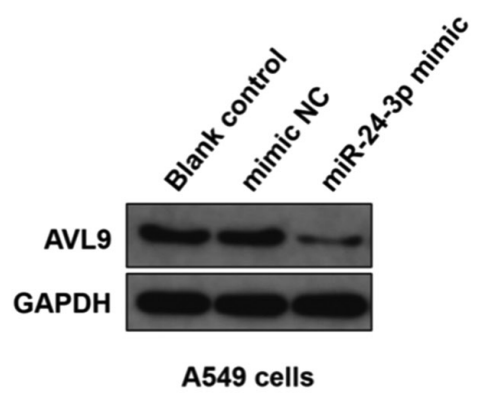

D miR-24-3p

AVL9 3'UTR
B

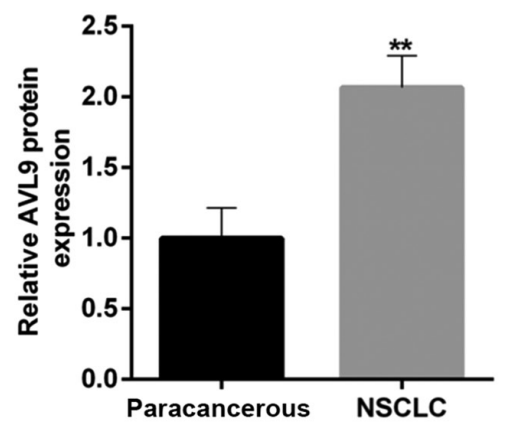

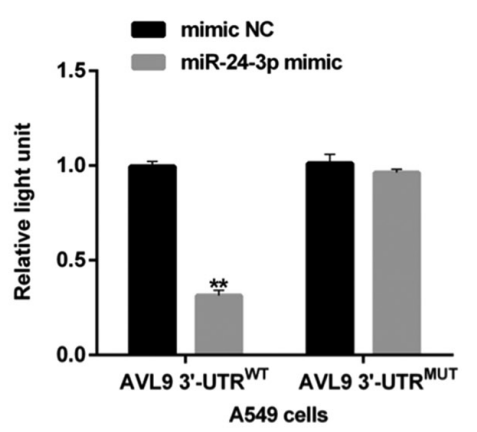

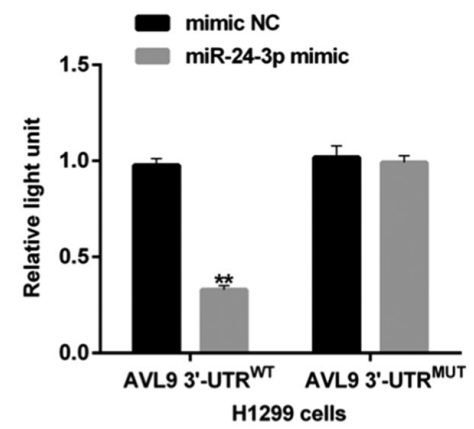

3' -GACAAGGACGACTTGACTCGGT- 5' 5'-TAC TTTTATTTCAGTCTGAGCCT- 3'

H1299 cells

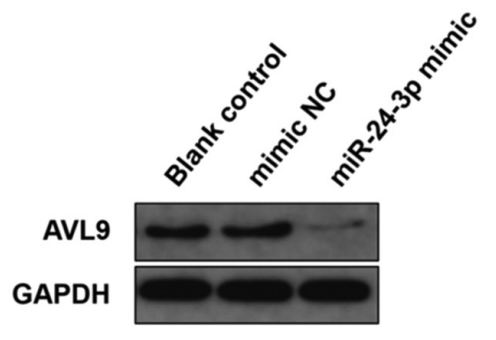

H1299 cells

Figure 9. Effects of miR-24-3p on protein expression of AV9L. (A) Protein expression level of AVL9 in human NSCLC tissues and paired-paracancerous tissues was determined via immunoblotting, ( $\mathrm{n}=20$ /group). (B) Semi-quantitative analysis of the protein expression level of AVL9 in panel A. (C) Protein expression level of AVL9 in NSCLC cells, including H292, A549, H1299 and H460, and HBE cells, as detected by western blotting. (D) The binding sites between miR-24-3p and circ_0058357 were identified and shown in the top part of this panel. A549 and H1299 cells were co-transfected with mimic NC/miR-24-3p mimic and WT/MUT-AVL9, then the luciferase activity of AVL9 was detected and normalized with Renilla. (E) A549 and H1299 cells were firstly transfected with mimic NC or miR-24-3p mimic. Then, the protein expression level of AVL9 was evaluated via western blotting in the two cell lines. ${ }^{* *} \mathrm{P}<0.01 . \mathrm{NC}$, negative control; miR, microRNA; AVL9, AVL9 cell migration associated; NSCLC, non-small cell lung cancer; WT, wild-type; MUT, mutant; UTR, untranslated region.

signaling axis in tumor tissues (Fig. 10A-C). In summary, it was suggested that AVL9 may be an important downstream switch of the circ_0058357/miR-24-3p axis in the process of tumorigenesis of NSCLC.

\section{Discussion}

To date, as a tumor driver gene, whether and how AVL9 contributes to the progression of NSCLC remains elusive. In the present study, it was demonstrated that AVL9 was possibly regulated by the circ_0058357/miR-24-3p signaling pathway, thereby leading to the development of NSCLC. The present data firstly indicated that the involvement of circ_0058357 could accelerate tumor progress of NSCLC via a sponge-regulation mechanism, thereby promoting the expression of AVL9.

Accumulating evidence has suggested that dysregulated circRNAs contribute to carcinogenesis and tumor progression. For instance, in comparison with healthy subjects, both hsa_circ_0014130 and hsa_circ_0020123 are upregulated in NSCLC tissues and have a significant positive correlation with TNM stage and lymph node metastasis, which demonstrates good diagnostic potential $(20,21)$. hsa_circ_0013958 can also 
A

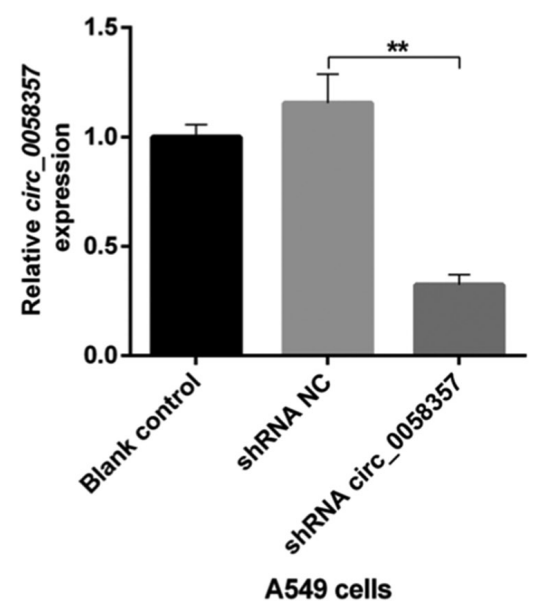

C

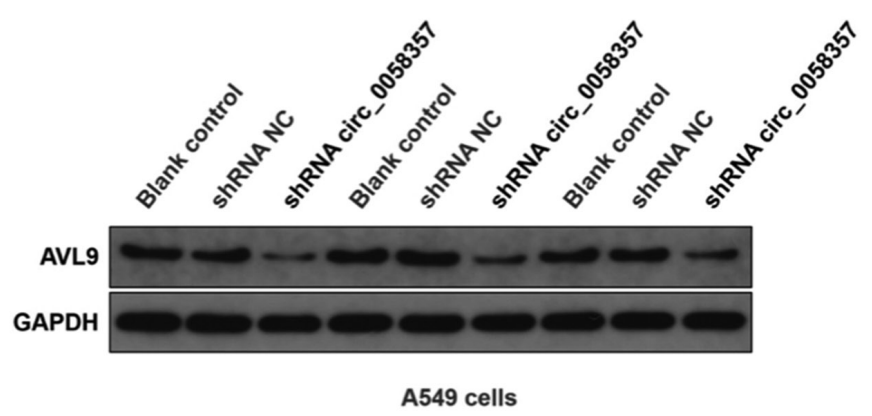

B
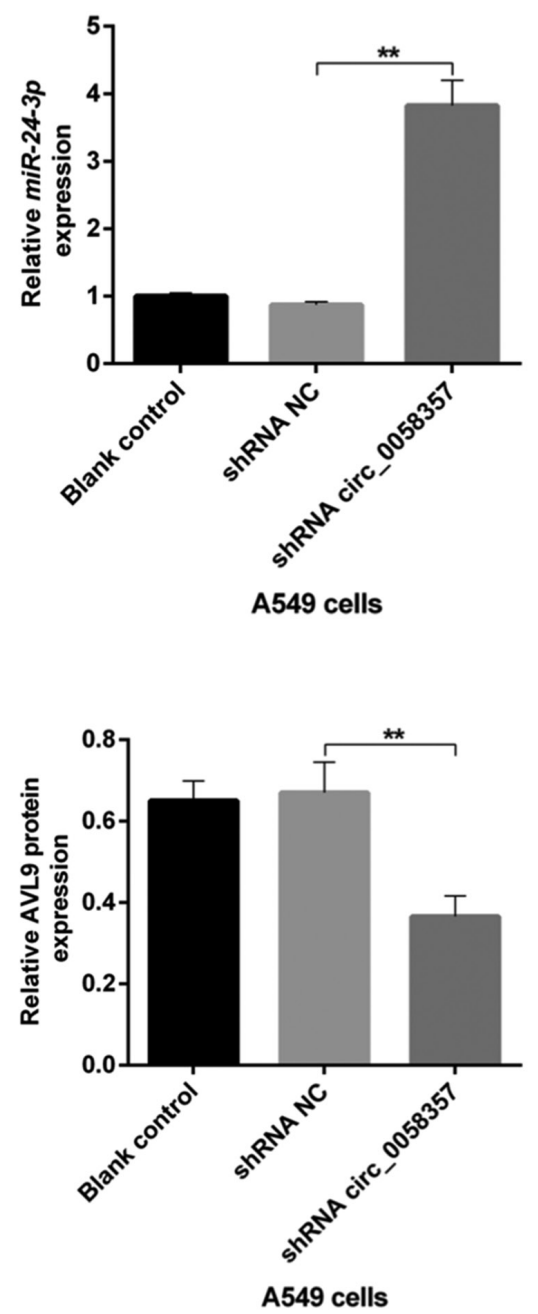

Figure 10. Analysis of the association between circ_0058357, miR-24-3p and AVL9 in mouse tumor tissues. A549 cells were transfected with shRNA NC and circ_0058357 shRNA. Then, A549 cells were injected into the right frank of mice to establish tumor xenograft model. After 7 weeks post-injection, tumor tissues were obtained and the relative expression levels of (A) circ_0058357 and (B) miR-24-3p were determined via reverse transcription-quantitative PCR. (C) Protein expression level and semi-quantitative analysis of AVL9 in tumor tissues, as determined via immunoblotting. ${ }^{* *} \mathrm{P}<0.01$. NC, negative control; miR, microRNA; AVL9, AVL9 cell migration associated; circ, circular RNA.

be considered as a potential non-invasive biomarker for the early diagnosis of patients with NSCLC (22). In the current study, circ_0058357 expression was notably enhanced in human NSCLC tissues, while it was lowly expressed in paracancerous tissues. However, a larger sample size is required to further investigate the application of circ_0058357 in diagnosing NSCLC. It has been confirmed that hsa_circ_0075930 is highly expressed in NSCLC, and its depletion impairs cell proliferation, migration and invasion potential by abolishing EMT progress (23). Moreover, knockdown of hsa_circ_0007385 markedly reduces tumor growth of NSCLC cells in vivo (24). These aberrant circRNAs may be cancer-promoting genes and could be potential therapeutic targets for NSCLC. In the present study, knockdown of circ_0058357 repressed cancer properties, including cell viability, cell migration and tumor growth in vitro and in vivo. These data suggested that circ_0058357 could be a novel oncogene of NSCLC.

Previous research has shown that hsa_circ_0008305 [produced from protein tyrosine kinase 2 (PTK2) gene, termed as circPTK2] inhibited TGF- $\beta$-induced EMT, and thus, blunts cancer metastasis by controlling TRIM24 in NSCLC (12). However, circPTK2 did not influence its parent gene PTK2 expression. Another previous study reported that exon-intron or intron-derived circRNAs increased the transcription of their parent gene, but exon-derived circRNAs do not affect the expression of their parent genes (25). However, the present study did not evaluate the regulatory role of circ_0058357 on its parent gene ATG9A. ATG9A is an important target to develop new specific cancer therapies, such as those for triple negative breast cancer and colorectal cancer $(26,27)$. Thus, if circ_0058357 can affect the expression of its parent $A T G 9 A$ gene, this finding would have high significance in the clinic.

While circRNAs have a low abundance, they are emerging as key oncogenic stimuli in cancer (28). circRNAs work as miRNA sponges (29). Most recently, circ_0002483 has been confirmed to regulate the expression levels of cancer-related genes by sponging miR-182-5p, thereby modulating NSCLC progression (30). Furthermore, hsa_circ_0004015 acts as a sponge for miR-1183 to regulate its target gene 
3-phosphoinositide dependent protein kinase 1 and exerts oncogenic effects in NSCLC (31). It has demonstrated that miR-24-3p may act as an oncogene by targeting chromodomain helicase DNA-binding domain 5 in head and neck squamous cell carcinoma and $\mathrm{p} 27^{\mathrm{Kip} 1}$ in breast cancer, respectively $(32,33)$. Moreover, miR-24-3p can be regulated by several lncRNAs in numerous types of cancer, including hepatocellular carcinoma (34), prostate cancer (35) and lung cancer (36). miR-24-3p is also sponged by circ_0080425 in diabetic nephropathy, leading to cell proliferation and fibrosis inhibition (37). However, the sponge regulation of miR-24-3p by circRNAs in NSCLC remains unknown. In the present study, it was observed that miR-24-3p was directly sponged by circ_0058357 in NSCLC cells. Moreover, exogenous miR-24-3p abolished the circ_0058357 OE-induced tumorigenesis of NSCLC cells. These findings suggested that circ_0058357-mediated carcinogenesis in NSCLC could be primarily via sponging miR-24-3p.

As aforementioned, AVL9 may be considered as an oncogene in colorectal cancer (16). Herein, it was discovered that AVL9 protein expression was higher in NSCLC tissues and cells compared with in paracancerous tissues and HBE cells, suggesting that AVL9 could also be a cancer-driven gene in NSCLC. AVL9 can be directly regulated by miRNA in cancer cells (17). The present study demonstrated that AVL9 was the direct downstream target of miR-24-3p in NSCLC cells. Additionally, in mouse tumor tissues, circ_0058357 knockdown mediated the decline of miR-24-3p, and subsequently, the increase of AVL9. It was indicated that circ_0058357 may positively regulate the expression of AVL9 by sponging miR-24-3p in NSCLC cells.

Except for in colorectal cancer, the AVL9 protein is also downregulated by lncRNA CRPAT4-siRNA, thereby inhibiting cell migration and proliferation in the absence of hypoxia-inducible factor $1 \alpha$ in clear cell renal cell carcinomas (38). Of note, AVL9 ablation notably suppresses the migration of A549 cells (39). Therefore, AVL9 may be the key downstream switch in the progress of the circ_0058357/miR-24-3p signaling axis-induced excessive cell proliferation, metastasis and tumor growth of NSCLC cells. Mechanistically, the generation of secretory vesicles in late secretory pathway serves a crucial role in the progression of several types of cancer, such as NSCLC, breast cancer and colorectal cancer $(40,41)$. Rab GTPases define the vesicle trafficking pathways underpinning cell polarization, proliferation and migration, which involves AVL9 $(39,42)$. As a pivotal regulatory factor in the late secretory pathway, it was suggested that the circ_0058357/miR-24-3p pathway-induced carcinogenicity in NSCLC cells by elevating AVL9 expression should be associated with vesicle secretion events. However, the link between hsa_circ_0058357 and hsa-miR-24-3p/AVL9 should be investigated using the combination of AVL9 OE plasmids and circ_0058357 shRNA in a xenograft model. Additionally, a larger sample size of specimens should be included in a future study.

In conclusion, the present study identified a novel circRNA, circ_0058357, as a potential therapeutic biomarker in patients with NSCLC. The functional experiments suggested the significant promotion role of circ_0058357 on cell migration, migration and tumor growth by positively regulating the expression level of AVL9 by sponging miR-24-3p. These findings encourage further efforts to study the detailed regulatory mechanisms underlying the circ_0058357/miR-24-3p/AVL9-mediated oncogenesis in NSCLC progression.

\section{Acknowledgements}

Not applicable.

\section{Funding}

This work was supported by the Projects of Health and Family Planning Commission of Zhejiang Province (grant no. 2018K Y600), Zhejiang Provincial Natural Science Foundation of China (grant no. LY21H010001), Zhejiang Provincial Science and Technology of Traditional Chinese Medicine (grant no. 2021ZB219), Medical and Health Planning Program of Zhejiang Province (grant no. 2020PY001), Health Innovation Talent of Zhejiang Province (grant no. 2021PY038) and Beijing Medical and Health Foundation (grant no. YWJKJJHKYJJ-F2056G).

\section{Availability of data and materials}

All data generated or analyzed during this study are included in this published article.

\section{Authors' contributions}

WF conceived the idea for the study. WF and LS drafted the manuscript. DW and LS designed and performed the CCK-8, Transwell, apoptosis and transfection assays. LS analyzed the data and designed the figures. DW contributed to xenograft and western blotting assays. WF provided the clinical breast cancer specimens. WF and LS confirm the authenticity of all the raw data. All authors discussed the results and edited this manuscript. All authors read and approved the final manuscript.

\section{Ethics approval and consent to participate}

All patient experiments were approved by the local Ethics Committee of Affiliated Hangzhou First People's Hospital (ethical approval no. 2016HZFPH-B072Z). All animal experiments were approved by the Animal Ethics Committee of The Affiliated Hangzhou First People's Hospital (approval no. 2016HZFPH-AB19Z). Signed informed consent was obtained from all the patients

\section{Patient consent for publication}

Not applicable.

\section{Competing interests}

The authors declare that they have no competing interests.

\section{References}

1. Zheng HM, Zhan YT, Liu SL, Lu J, Luo J, Feng J and Fan S: The roles of tumor-derived exosomes in non-small cell lung cancer and their clinical implications. J Exp Clin Canc Res 37: 226, 2018. 
2. Siegel RL, Miller KD and Jemal A: Cancer statistics, 2020. CA Cancer J Clin 70: 7-30, 2020.

3. Liu G, Pei F, Yang F, Li L, Amin AD, Liu S, Buchan JR and Cho WC: Role of autophagy and apoptosis in non-small-cell lung cancer. Int J Mol Sci 18: 367, 2017.

4. Rong B, Nan Y, Liu H and Gao W: Increased stathmin correlates with advanced stage and poor survival of non-small cell lung cancer. Cancer Biomark 19: 35-43, 2017.

5. Liu ZL, Zhu WR, Zhou WC, Ying HF, Zheng L, Guo YB Chen JX and Shen XH: Traditional Chinese medicinal herbs combined with epidermal growth factor receptor tyrosine kinase inhibitor for advanced non-small cell lung cancer: A systematic review and meta-analysis. J Integr Med 12: 346-358, 2014.

6. Gupta GP and Massague J: Cancer metastasis: Building a framework. Cell 127: 679-695, 2006.

7. Cui J, Li W, Liu G, Chen X, Gao X, Lu H and Lin D: A novel circular RNA, hsa_circ_0043278, acts as a potential biomarker and promotes non-small cell lung cancer cell proliferation and migration by regulating miR-520f. Artif Cells Nanomed Biotechnol 47: 810-821, 2019.

8. Xie G: Circular RNA hsa-circ-0012129 promotes cell proliferation and invasion in 30 cases of human glioma and human glioma cell lines U373, A172, and SHG44, by targeting MicroRNA-661 (miR-661). Med Sci Monit 24: 2497-2507, 2018.

9. Zhang Z, Yu K, Liu O, Xiong Y, Yang X, Wang S, Zhang S, Feng Y and Peng Y: Expression profile and bioinformatics analyses of circular RNAs in keloid and normal dermal fibroblasts. Exp Cell Res 388: 111799, 2020.

10. Yu W, Jiang H, Zhang H and Li J: Hsa_circ_0003998 promotes cell proliferation and invasion by targeting miR-326 in non-small cell lung cancer. Onco Targets Ther 11: 5569-5577, 2018.

11. Liu C, Zhang Z and Qi D: Circular RNA hsa_circ_0023404 promotes proliferation, migration and invasion in non-small cell lung cancer by regulating miR-217/ZEB1 axis. Onco Targets Ther 12: 6181-6189, 2019.

12. Wang L, Tong X, Zhou Z, Wang S, Lei Z, Zhang T, Liu Z, Zeng Y, Li C, Zhao J, et al: Circular RNA hsa_circ_0008305 (circPTK2) inhibits TGF- $\beta$-induced epithelial-mesenchymal transition and metastasis by controlling TIF $1 \gamma$ in non-small cell lung cancer. Mol Cancer 17: 140, 2018.

13. Zhang L, Nebane NM, Wennerberg K, Li Y, Neubauer V, Hobrath JV, McKellip S, Rasmussen L, Shindo N, Sosa M, et al: A high-throughput screen for chemical inhibitors of exocytic transport in yeast. Chembiochem 11: 1291-1301, 2010.

14. Harsay E and Schekman R: Avl9p, a member of a novel protein superfamily, functions in the late secretory pathway. Mol Biol Cell 18: 1203-1219, 2007.

15. Li Y, Xu J, Xiong H, Ma Z, Wang Z, Kipreos ET, Dalton S and Zhao S: Cancer driver candidate genes AVL9, DENND5A and NUPL1 contribute to MDCK cystogenesis. Oncoscience 1: 854-865, 2014

16. Tang J, Li Y, Lyon K, Camps J, Dalton S, Ried T and Zhao S: Cancer driver-passenger distinction via sporadic human and dog cancer comparison: A proof-of-principle study with colorectal cancer. Oncogene 33: 814-822, 2014

17. Wang $\mathrm{H}$, Yu M, Hu W, Chen X, Luo Y, Lin X, Zeng Y and Yao X: Linc00662 promotes tumorigenesis and progression by regulating miR-497-5p/AVL9 axis in colorectal cancer. Front Genet 10: 1385, 2020.

18. Livak KJ and Schmittgen TD: Analysis of relative gene expression data using real-time quantitative PCR and the 2(-Delta Delta C(T)). Methods 25: 402-408, 2001.

19. Olbromski M, Rzechonek A, Grzegrzolka J, Glatzel-Plucinska N, Chachaj A, Werynska B, Podhorska-Okolow M and Dziegiel P: Influence of miR-7a and miR-24-3p on the SOX18 transcript in lung adenocarcinoma. Oncol Rep 39: 201-208, 2018.

20. Zhang S, Zeng X, Ding T, Guo L, Li Y, Ou S and Yuan H: Microarray profile of circular RNAs identifies hsa_circ 0014130 as a new circular RNA biomarker in non-small cell lung cancer. Sci Rep 8: 2878, 2018

21. Qu D, Yan B, Xin R and Ma T: A novel circular RNA hsa circ_0020123 exerts oncogenic properties through suppression of miR-144 in non-small cell lung cancer. Am J Cancer Res 8 : $1387-1402,2018$

22. Zhu X, Wang X, Wei S, Chen Y, Chen Y, Fan X, Han S and Wu G: hsa circ 0013958: A circular RNA and potential novel biomarker for lung adenocarcinoma. FEBS J 284: 2170-2182, 2017.

23. Li J, Wang J, Chen Z, Chen Y and Jin M: Hsa circ 0079530 promotes cell proliferation and invasion in non-small cell lung cancer. Gene 665: 1-5, 2018.
24. Jiang MM, Mai ZT, Wan SZ, Chi YM,Zhang X, Sun BH and Di QG Microarray profiles reveal that circular RNA hsa_circ_0007385 functions as an oncogene in non-small cell lung cancer tumorigenesis. J Cancer Res Clin Oncol 144: 667-674, 2018.

25. Cortés-López M and Miura P: Emerging functions of circular RNAs. Yale J Biol Med 89: 527-537, 2016.

26. Gil J, Ramsey D, Pawlowski P, Szmida E, Leszczynski P, Bebenek $M$ and Sasiadek MM: Interdependence between an expression of the ATG9A gene and the BAX gene in colorectal cancer. J Biol Regul Homeost Agents 33: 183-185, 2019.

27. Claude-Taupin A, Fonderflick L, Gauthier T, Mansi L, Pallandre JR, Borg C, Perez V, Monnien F, Algros MP, Vigneron $\mathrm{M}$, et al: ATG9A is overexpressed in triple negative breast cancer and its in vitro extinction leads to the inhibition of pro-cancer phenotypes. Cells 7: 248, 2018.

28. Lyu DB and Huang SL: The emerging role and clinical implication of human exonic circular RNA. Rna Biol 14: 1000-1006, 2017.

29. Kulcheski FR, Christoff AP and Margis R: Circular RNAs are miRNA sponges and can be used as a new class of biomarker. J Biotechnol 238: 42-51, 2016.

30. Li X, Yang B, Ren H, Xiao T, Zhang L, Li L, Li M, Wang X, Zhou H and Zhang W: Hsa_circ_0002483 inhibited the progression and enhanced the Taxol sensitivity of non-small cell lung cancer by targeting miR-182-5p. Cell Death Dis 10: 953, 2019.

31. Zhou Y, Zheng X, Xu B, Chen L, Wang Q, Deng H and Jiang J: Circular RNA hsa_circ_0004015 regulates the proliferation, invasion, and TKI drug resistance of non-small cell lung cancer by miR-1183/PDPK1 signaling pathway. Biochem Biophys Res Commun 508: 527-535, 2019.

32. Sun X, Xiao D, Xu T and Yuan Y: miRNA-24-3p promotes cell proliferation and regulates chemosensitivity in head and neck squamous cell carcinoma by targeting CHD5. Future Oncol 12: 2701-2712, 2016

33. Lu K, Wang J, Song Y, Zhao S, Liu H, Tang D, Pan B, Zhao H and Zhang Q: miRNA-24-3p promotes cell proliferation and inhibits apoptosis in human breast cancer by targeting p27Kip1. Oncol Rep 34: 995-1002, 2015.

34. Fan JC, Zeng F, Le YG and Xin L: LncRNA CASC2 inhibited the viability and induced the apoptosis of hepatocellular carcinoma cells through regulating miR-24-3p. J Cell Biochem 119: 6391-6397, 2018

35. Li X, Han X, Wei P, Yang J and Sun J: Knockdown of lncRNA CCAT1 enhances sensitivity of paclitaxel in prostate cancer via regulating miR-24-3p and FSCN1. Cancer Biol Ther 21: 452-462, 2020.

36. Su C, Shi K, Cheng X, Han Y,Li Y, Yu D and Liu Z: Long noncoding RNA LINC00472 inhibits proliferation and promotes apoptosis of lung adenocarcinoma cells via regulating miR-24-3p/DEDD. Technol Cancer Res Treat 17: 1533033818790490, 2018.

37. Liu H, Wang X, Wang ZY and Li L: Circ 0080425 inhibits cell proliferation and fibrosis in diabetic nephropathy via sponging miR-24-3p and targeting fibroblast growth factor 11. J Cell Physiol 235: 4520-4529, 2020.

38. Zhang W, Wang J, Chai R, Zhong G, Zhang C, Cao W, Yan L, Zhang $\mathrm{X}$ and $\mathrm{Xu} \mathrm{Z}$ : Hypoxia-regulated lncRNA CRPAT4 promotes cell migration via regulating AVL9 in clear cell renal cell carcinomas. Onco Targets Ther 11: 4537-4545, 2018.

39. Linford A, Yoshimura S, Nunes Bastos R, Langemeyer L, Gerondopoulos A, Rigden DJ and Barr FA: Rab14 and its exchange factor FAM116 link endocytic recycling and adherens junction stability in migrating cells. Dev Cell 22: 952-966, 2012.

40. Wang $M$ and Petersen NO: Lipid-coated gold nanoparticles promote lamellar body formation in A549 cells. Biochim Biophys Acta 1831: 1089-1097, 2013.

41. Schaaij-Visser TB, de Wit M, Lam SW and Jiménez CR: The cancer secretome, current status and opportunities in the lung, breast and colorectal cancer context. Biochim Biophys Acta 1834: 2242-2258, 2013

42. Zhang L, Huang M and Harsay E: A chemical genetic screen for modulators of exocytic transport identifies inhibitors of a transport mechanism linked to GTR2 function. Eukaryot Cell 9: 116-126, 2010.

This work is licensed under a Creative Commons Attribution-NonCommercial-NoDerivatives 4.0 International (CC BY-NC-ND 4.0) License. 